\title{
The 3-D ionization structure of the planetary nebula NGC $6565^{\star}$
}

\author{
M. Turatto ${ }^{1}$, E. Cappellaro ${ }^{1}$, R. Ragazzoni ${ }^{1}$, S. Benetti $^{1}$, and F. Sabbadin ${ }^{1}$ \\ Osservatorio Astronomico di Padova, vicolo dell'Osservatorio 5, 35122 Padova, Italy
}

Received 26 November 2001 / Accepted 21 January 2002

\begin{abstract}
A detailed study of the planetary nebula NGC 6565 has been carried out on long-slit echellograms $(\lambda / \Delta \lambda=60000$, spectral range $=\lambda \lambda 3900-7750 \AA)$ at six, equally spaced position angles. The expansion velocity field, the $\mathrm{c}(\mathrm{H} \beta)$ distribution and the radial profile of the physical conditions (electron temperature and density) are obtained. The distance, radius, mass and filling factor of the nebula and the temperature and luminosity of the central star are derived. The radial ionization structure is analyzed using both the classical method and the photo-ionization code CLOUDY. Moreover, we present the spatial structure in a series of images from different directions, allowing the reader to "see" the nebula in 3-D. NGC 6565 results to be a young (2000-2500 years), patchy, optically thick triaxial ellipsoid ( $a=10.1 \mathrm{arcsec}, a / b=1.4, a / c=1.7)$ projected almost pole-on. The matter close to major axis was swept-up by some accelerating agent (fast wind? ionization? magnetic fields?), forming two faint and asymmetric polar cups. A large cocoon of almost neutral gas completely embeds the ionized nebula. NGC 6565 is in a recombination phase, because of the luminosity drop of the massive powering star, which is reaching the white dwarf domain $\left(\log T_{*} \simeq 5.08 \mathrm{~K} ; \log L_{*} / L_{\odot} \simeq 2.0\right)$. The stellar decline started about 1000 years ago, but the main nebula remained optically thin for other 600 years before the recombination phase occurred. In the near future the ionization front will re-grow, since the dilution factor due to the expansion will prevail on the slower and slower stellar decline. NGC 6565 is at a distance of $2.0( \pm 0.5) \mathrm{kpc}$ and can be divided into three radial zones: the "fully ionized" one, extending up to $0.029-0.035 \mathrm{pc}$ at the equator $(0.050 \mathrm{pc}$ at the poles), the "transition" one, up to $0.048-0.054 \mathrm{pc}(0.080 \mathrm{pc})$, the "halo", detectable up to $0.110 \mathrm{pc}$. The ionized mass $\left(\simeq 0.03 M_{\odot}\right)$ is only a fraction of the total mass $\left(\geq 0.15 M_{\odot}\right)$, which has been ejected by an equatorial enhanced superwind of $4( \pm 2) \times 10^{-5} M_{\odot} \mathrm{yr}^{-1}$ lasted for $4( \pm 2) \times 10^{3}$ years.
\end{abstract}

Key words. planetary nebulae: individual: NGC 6565 - ISM: kinematics and dynamics

\section{Introduction}

The late evolution of low and intermediate mass stars $\left(1.0 M_{\odot}<M_{*}<8.0 M_{\odot}\right)$ is characterized by the planetary nebula $(\mathrm{PN})$ metamorphosis: an asymptotic giant branch (AGB) star gently pushes out the surface layers in a florilegium of forms, crosses the HR diagram and reaches the white dwarf regime (Aller 1984; Pottasch 1984; Osterbrock 1989).

So far the interpretation of the exuberant morphologies exhibited by the $\mathrm{PNe}$ in terms of detailed

\footnotetext{
Send offprint requests to: M. Turatto,

e-mail: turatto@pd.astro.it

* Based on observations made with ESO Telescopes at the La Silla Observatories, under programme ID 65.I-0524, and on observations made with the NASA/ESA Hubble Space Telescope, obtained from the data archive at the Space Telescope Institute (observing program GO 7501; P.I. Arsen Hajian). STScI is operated by the association of Universities for Research in Astronomy, Inc. under the NASA contract NAS 5-26555. We have applied the photoionization code CLOUDY, developed at the Institute of Astronomy of the Cambridge University.
}

three-dimensional structures and physical conditions of the ionized gas was limited by projection effects, leading to approximate spatial forms and to unrealistic assumptions for the main parameters, e.g. electron temperature and electron density constant all over the nebula (Aller 1984, 1990, 1994).

A 3-D reconstruction technique for studying at large and small scales the morphology, physical conditions, ionization, spatial structure and evolutionary status of PNe has been introduced by Sabbadin et al. (2000a,b) and Ragazzoni et al. (2001), based on echellograms of moderate spectral resolution $(R \sim 22000-25000)$. The key of the 3-D methodology is simple: the $\mathrm{PN}$ is an expanding plasma. Thus the position, thickness and density of each elementary volume can be derived from the radial velocity, width and flux of the corresponding emission.

The procedure has been applied to:

- NGC 40: an optically thick, very low excitation barrelshaped nebula with thin arcs emerging at both ends of the major axis, powered by a luminous and "cold" WC8 star presenting a large mass-loss rate. The fast, hydrogen depleted photospheric material ejected by the 

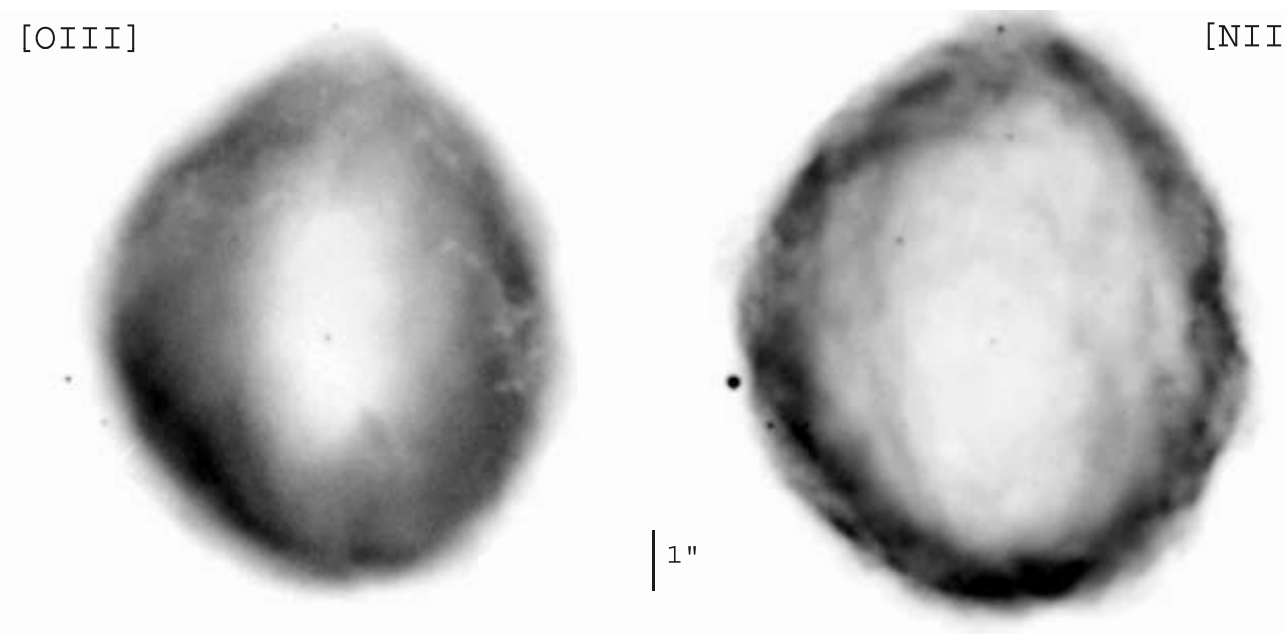

Fig. 1. WFPC2 appearance of NGC 6565 in [OIII] (left) and [NII] (right). The images have been retrieved from the HST archive. North is up and East is to the left.

nucleus is gradually modifying the chemical composition of the innermost nebular regions (Sabbadin et al. 2000a);

- NGC 1501: an evolved, high excitation, optically thin oblate ellipsoid, denser in the equatorial belt, deformed by several bumps, embedded in a homogeneous, inwards extended cocoon and ionized by a "hot" and luminous WC4 star exhibiting nonradial $g$-mode pulsations (Sabbadin et al. 2000b; Ragazzoni et al. 2001).

In order to deepen the analysis, we have started a survey at high spectral and spatial resolutions with the ESO NTT. The superb quality of this material allows us to study at unprecedented accuracies objects with different morphology, e.g. NGC 7009 (the "Saturn" nebula), the tetra-lobed IC 4634, the butterfly $\mathrm{HB} 5, \mathrm{Mz} 3$ and NGC 6537, the double-envelope NGC 5882, NGC 6153 and NGC 6818.

To simplify the application of the $3-\mathrm{D}$ method we decided to begin with an "easy" nebula without FLIERS (fast, low ionization emitting regions), BRETS (bipolar, rotating, episodic jets), ansae, wings, multiple envelopes etc., and naively selected NGC 6565.

\section{The nebula}

The bright, compact PN NGC 6565 (PNG 003.5-04.6, Acker et al. 1992) is "a minute oval ring $10^{\prime \prime} \times 8^{\prime \prime}$ in PA about $5^{\circ}$. Considerably fainter along the major axis, and the center is relatively vacant" (Curtis 1918). Its HST appearance is shown in Fig. 1: the [OIII] emission forms a quite homogeneous oval ring, sprinkled by a number of dark globules and spikes, mainly in the Northern sector. It is surrounded by a sharp, patchy [NII] layer presenting two faint lobes in $\mathrm{PA} \simeq 145^{\circ}$. Note also the light $\mathrm{N}-\mathrm{S}$ asymmetry of the nebula with respect to the (barely visible) central star. The large stratification effects and the external, faint, low ionization regions are better seen in Fig. 2, presenting the $[\mathrm{NII}] /[\mathrm{OIII}]$ distribution.

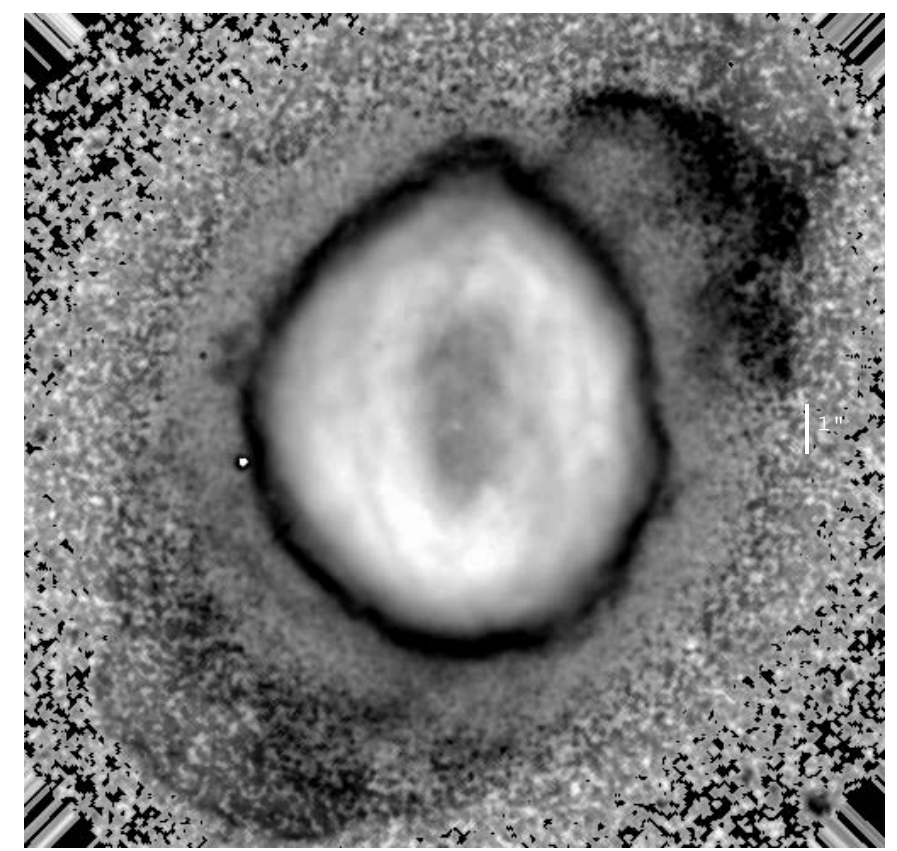

Fig. 2. $[\mathrm{NII}] /[\mathrm{OIII}]$ distribution over NGC 6565 (original frames and orientation as in Fig. 1), showing the large stratification effects and the faint, low ionization regions protruding from the main nebula in $\mathrm{PA} \simeq 145^{\circ}$.

NGC 6565 appears peculiar in many respects:

- despite the simple elliptical shape (Stanghellini et al. 1993; Gorny et al. 1997), it shows a rich low ionization spectrum typical of bipolar PNe (Greig 1972) and is N overabundant (Aller et al. 1988);

- the infrared and radio excesses suggest the presence of a large amount of dusty neutral gas causing a detectable absorption of the optical radiation (Gathier et al. 1986; Stasinska et al. 1992); 
- in spite of the high surface brightness of the nebula, indicative of an early evolutionary phase, the central star is faint and hot, i.e. it is in, or close to, the white dwarf domain. Following Tylenda (1986), NGC 6565 could be in a recombination phase, due to the recent luminosity drop of the powering star.

Although the nebula is sometimes listed among the distance calibrators (Pottasch 1984; Sabbadin 1986; Gathier 1987; Zhang 1995), its distance is still poorly defined and the distance-extinction law gives contradictory results (Gathier et al. 1986; Maciel et al. 1986; de Oliveira-Abans \& Faundez-Abans 1991).

This paper is structured as follows: Sect. 3 presents the observational material and the reduction procedure, Sect. 4 is dedicated to the gas kinematics, Sect. 5 contains the $\mathrm{H} \alpha / \mathrm{H} \beta$ spectral maps, Sect. 6 analyses the radial distribution of the physical conditions (electron temperature and electron density), in Sect. 7 we derive the distance, the nebular mass and the central star parameters, in Sect. 8 the radial ionization structure and the distribution of the gas (ionized and neutral) are presented, Sect. 9 concerns the application of the photo-ionization model (CLOUDY), Sect. 10 describes the 3-D structure of the nebula in different ions, a short discussion and the conclusions are presented in Sect. 11.

\section{The observational material and reduction technique}

Six long-slit echellograms of NGC 6565 (exposure time $900 \mathrm{~s}$; spectral resolution 60000 with a slit 1.0 arcsec wide) have been obtained on July 28, 2000 at the ESO NTT equipped with EMMI in REMD mode using grating \#14, grism \#3 and CCD \#36 (spectral range $\lambda \lambda 3900-7750 \AA$, mean spatial scale along the slit 0.27 arcsec pix ${ }^{-1}$ ). During the observations the sky transparency was excellent and the seeing fluctuated between 0.53 and 0.89 arcsec. The 30 arcsec long slit was centered on the nebula for all the selected $\mathrm{PA}$, ranging from $0^{\circ}$ to $150^{\circ}$ with a constant step of $30^{\circ}$.

The reduction method follows conceptually the standard procedure, including bias, flat field, distortion correction and wavelength and flux calibration.

The slit length, much longer than the order separation, leads to a considerable superposition of the echelle orders. This, on the one hand does not affect the emission line spectrum of NGC 6565, on the other hand it does the flat field exposures. Thus, we were forced to apply a zeroorder correction using a white-light flat field. Thanks to the excellent CCD uniformity, the pixel to pixel sensitivity fluctuation left by this procedure is below $5 \%$ (verified on the night sky and the comparison spectrum lines). All things considered, it is a modest price to pay for keeping the whole spatial information of the PN emissions in the $\lambda \lambda 3900-7750 \AA$ spectral range.

After bias and flat field correction, the orders containing one or more emissions of physical interest were extracted and treated as single, long-slit spectra. The distortion correction and the wavelength calibration were performed by means of Th-Ar lamp exposures. During the frame registration, the various orders were scaled to the slit height at $\mathrm{H} \alpha$, chosen as reference line. Echellograms of the spectrophotometric standard star HR5501 (sampled at several positions along each order) were used to obtain the flux calibration.

We stress the substantial difference between our observational procedure and the one generally adopted, which introduces an interference filter to isolate a single order. On the contrary, we cover the whole $\lambda \lambda 3900-7750 \AA$ spectral range with a single exposure.

For illustrative purposes, in Fig. 3 we present the main part of the NTT+EMMI frame of NGC 6565 at PA = $150^{\circ}$, which includes the external, low ionization regions, and in Fig. 4 the detailed structure (at the same PA) of $\lambda 4686 \AA$ of HeII, $\lambda 5007 \AA$ of [OIII], $\lambda 5876 \AA$ of HeI, $\lambda 6300 \AA$ of $[\mathrm{OI}], \lambda 6563 \AA$ of HI, $\lambda 6584 \AA$ of $[\mathrm{NII}], \lambda 6731 \AA$ of [SII] and $\lambda 7135 \AA$ of [ArIII]. They suggest that the faint, low ionization regions visible in Fig. 2 are the projection of extended cups protruding from the main nebula. Note also the large stratification effects, the $[\mathrm{OI}]$ rises in the outermost parts and, in spite of the blurred $\mathrm{H} \alpha$ appearance due to thermal motions plus expansion velocity gradient plus fine structure, the similarity of the HI and [OIII] distributions. Following Williams (1973), these features are indicative of an optically thick nebula powered by a high temperature star.

A first, qualitative picture of the NGC 6565 structure can already be inferred from Figs. 1 to 4: the object is an ellipsoid with extended polar cups, projected almost pole-on. The possible optical appearance of the nebula seen equatorial-on is illustrated in Fig. 5, showing the PN NGC 6886 in [OIII] and [NII]. NGC 6565 and NGC 6886 are almost twins in many respects: nebular spectrum, physical conditions, ionization structure, evolutionary phase, luminosity and temperature of the central star. Likely, they derive from similar progenitors.

\section{The gas kinematics}

We introduce two important definitions relative to each emission line, which will be extensively used in the paper (they are shown in the lower-right panel of Fig. 4):

1) the zero-velocity pixel column (zvpc, see Sabbadin et al. 2000a, 2000b; Ragazzoni et al. 2001), corresponding to the recession velocity of the whole nebula, collects the photons of the ionized gas expanding perpendicularly to the line of sight;

2) the central star pixel line (cspl), parallel to the dispersion, represents the nebular material projected at the apparent position of the star, whose motion is purely radial.

In a certain sense the zvpc and the cspl are complementary: the first is independent on the expansion velocity and gives only spatial information, the second measures 


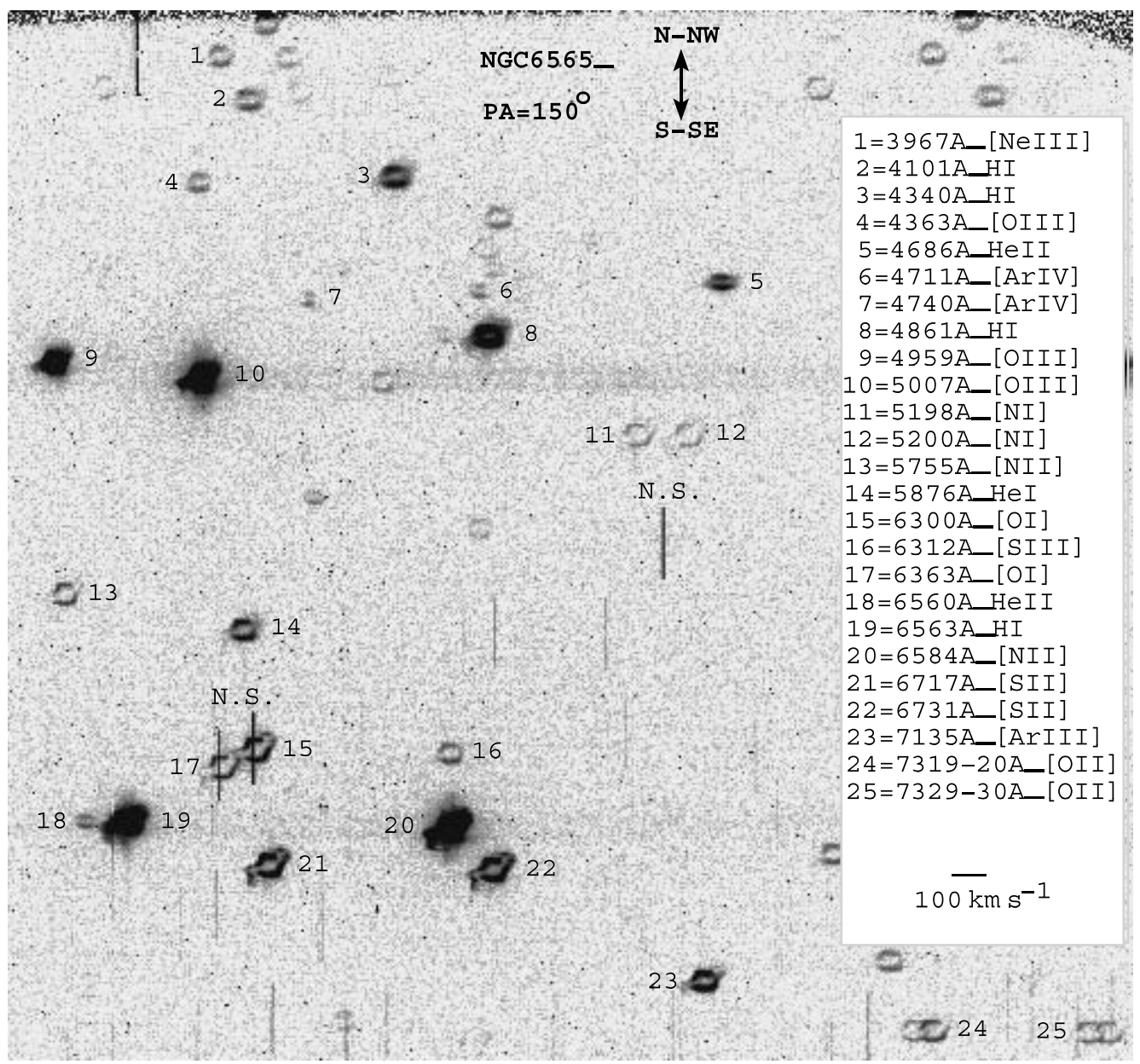

Fig. 3. Main part of the NTT+EMMI frame of NGC 6565 at $\mathrm{PA}=150^{\circ}$ (in logarithmic scale); the principal nebular emissions are identified and the strongest night sky lines (N.S.) indicated. The spatial scale is given by the N.S. extension, corresponding to the slit length of $30^{\prime \prime}$.
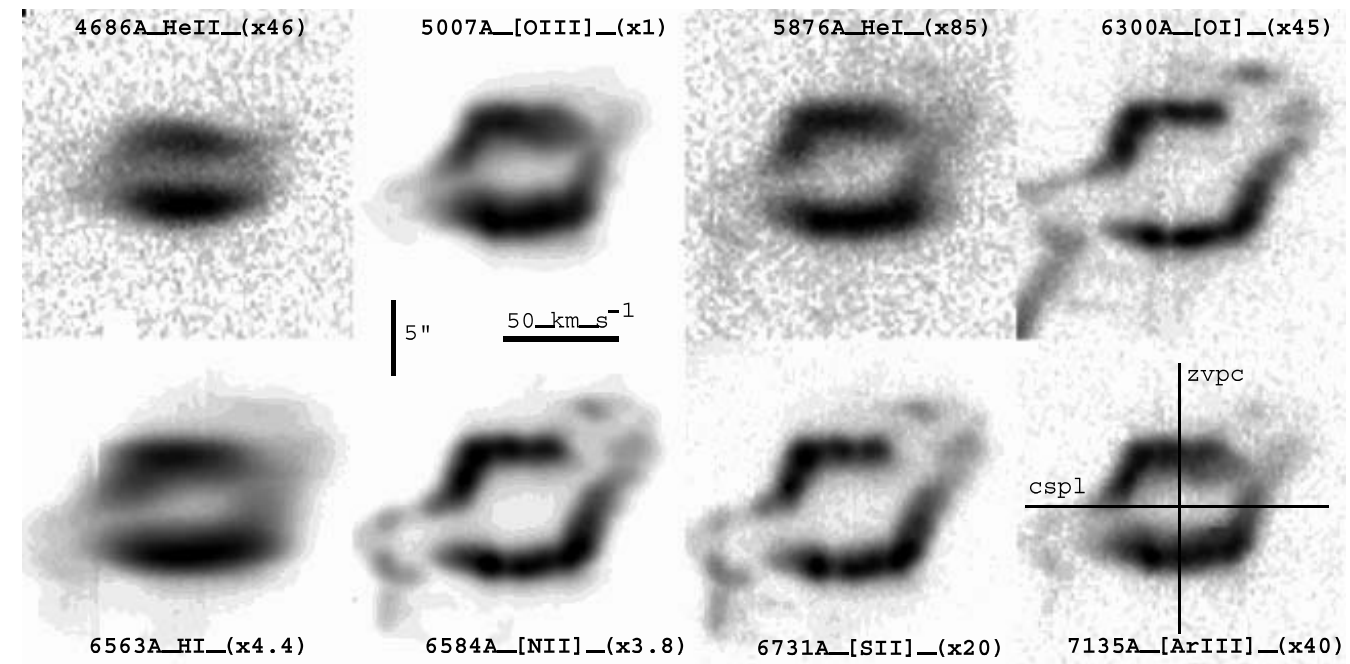

Fig. 4. Detailed structure (in logarithmic scale) of some representative emissions observed in NGC 6565 at $\mathrm{PA}=150^{\circ}$. The orientation is as in Fig. 3. The fluxes are multiplied for the factor given in parenthesis to render each emission comparable with $\lambda 5007 \AA$ of $[\mathrm{OIII}]$. The $\lambda 6300.304 \AA$ night sky line was removed from the [OI] nebular emission. Part of the [OI] $\lambda 6363 \AA$ emission (echelle order 96) appears as a moustache in the lower-left corner of the $\lambda 6300 \AA$ image (echelle order 97). The fast, blue-shifted wisp in the lower-left corner of the [NII] and [SII] frames is real. A hot pixel column grazes the H $\alpha$ emission. The zero-velocity pixel column (zvpc) and the central star pixel line (cspl), defined in Sect. 4, are shown in the lower-right panel. 


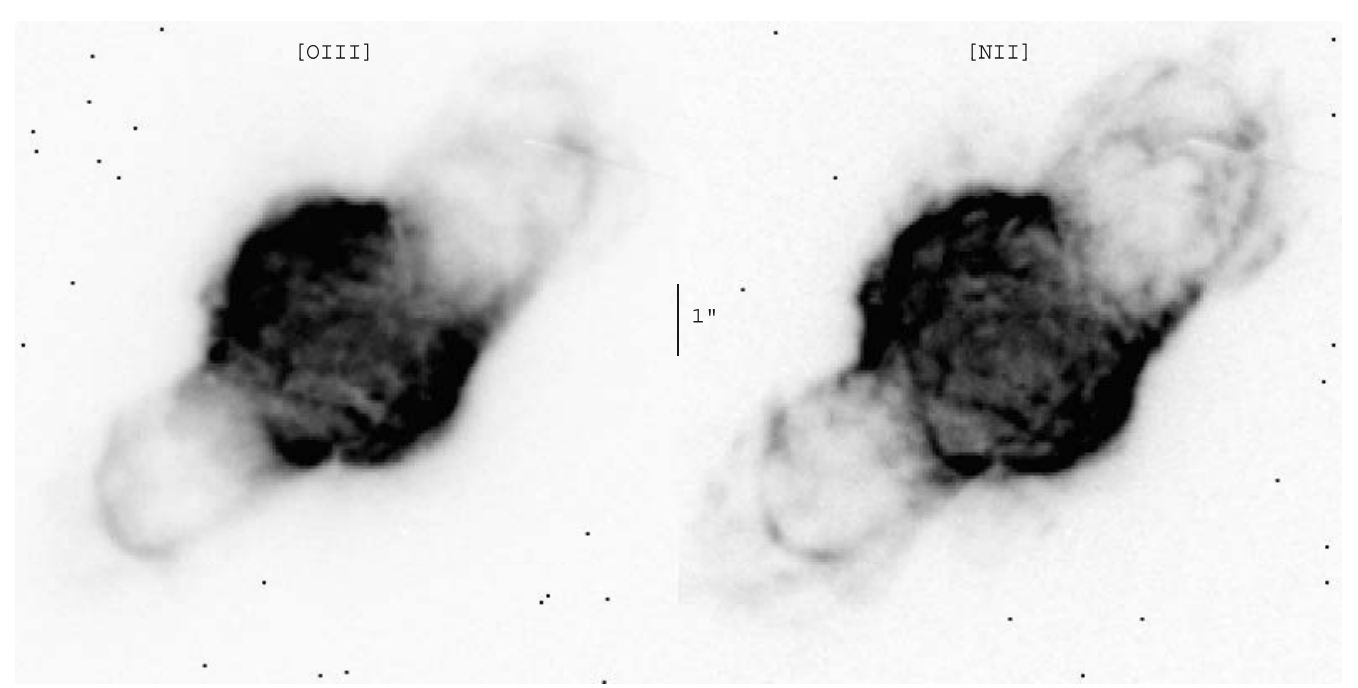

Fig. 5. HST imaging of the PN NGC6886 in [OIII] and [NII], showing the possible appearance of NGC 6565 seen almost equatorial-on.

Table 1. Expansion velocity in the cspl and peak separation in the zvpc for NGC 6565.

\begin{tabular}{|c|c|c|c|c|c|c|c|c|c|c|}
\hline \multirow[t]{2}{*}{ Ion } & \multirow{2}{*}{$\begin{array}{c}\lambda \\
(\AA)\end{array}$} & \multirow{2}{*}{$\begin{array}{l}\text { I.P. } \\
(\mathrm{eV})\end{array}$} & \multirow{2}{*}{$\frac{2 V_{\exp }(\text { main })}{\left(\mathrm{km} \mathrm{s}^{-1}\right)}$} & \multirow{2}{*}{$\begin{array}{l}V_{\exp }(\operatorname{cup}) \\
\left(\mathrm{km} \mathrm{s}^{-1}\right)\end{array}$} & \multicolumn{6}{|c|}{ peak separation $\left({ }^{\prime \prime}\right)$} \\
\hline & & & & & $\mathrm{PA}=0^{\circ}$ & $\mathrm{PA}=30^{\circ}$ & $\mathrm{PA}=60^{\circ}$ & $\mathrm{PA}=90^{\circ}$ & $\mathrm{PA}=120^{\circ}$ & $\mathrm{PA}=150^{\circ}$ \\
\hline$[\mathrm{NI}]$ & 5198 & 0.0 & 70.9: & - & 8.7: & 8.4: & 8.0: & $7.6:$ & $8.0:$ & 8.3: \\
\hline$[\mathrm{OI}]$ & 6300 & 0.0 & 67.7 & 55 & 8.7 & 8.4 & 8.0 & 7.6 & 8.0 & 8.3 \\
\hline [SII] & 6731 & 10.4 & 65.0 & 54 & 8.5 & 7.8 & 7.5 & 7.3 & 7.5 & 7.9 \\
\hline [OII] & 7319 & 13.6 & 59.4: & - & 7.8: & 7.7: & 7.2 & 7.0 & 7.4 & 7.8: \\
\hline $\mathrm{HI}$ & 6563 & 13.6 & 55.8 & 48: & 7.1 & 6.7 & 6.2 & 6.0 & 6.2 & 6.5 \\
\hline$[\mathrm{NII}]$ & 6584 & 14.5 & 60.6 & 54 & 8.2 & 7.6 & 7.4 & 7.1 & 7.4 & 7.8 \\
\hline [SIII] & 6312 & 23.4 & 57.5 & - & 7.6 & 6.9 & 6.7 & 6.3 & 6.5 & 6.7 \\
\hline [ClIII] & 5537 & 23.8 & $57.9:$ & - & 7.0: & 6.9: & 6.5: & 6.1: & 6.3: & 6.7: \\
\hline $\mathrm{HeI}$ & 5876 & 24.6 & 58.7 & 52: & 7.5 & 6.8 & 6.5 & 6.2 & 6.5 & 6.8 \\
\hline [ArIII] & 7135 & 27.6 & 57.2 & 50 & 7.3 & 7.0 & 6.8 & 6.3 & 6.5 & 7.0 \\
\hline [OIII] & 5007 & 35.1 & 56.7 & 49 & 6.9 & 6.4 & 6.3 & 6.0 & 6.3 & 6.4 \\
\hline [ArIV] & 4740 & 40.7 & 32.4 & - & 3.9 & 3.6 & 3.5 & 3.5 & 3.6 & 3.9 \\
\hline [NeIII] & 3967 & 41.0 & 55.2 & - & 7.2 & 6.6 & 6.5 & 6.3 & 6.6 & 6.6 \\
\hline HeII & 4686 & 54.4 & 44.2: & 42: & 4.5 & 3.5: & 3.8: & 3.7 & 4.0 & 4.1 \\
\hline$[\mathrm{ArV}]$ & 7005 & 59.8 & 17.0: & - & - & 3.4: & 3.2 : & 2.7: & 3.0: & 2.7: \\
\hline
\end{tabular}

the velocity field and contains only kinematical information. By combining the zvpc and the cspl we will derive the overall spatio-kinematical properties of the nebula.

The expansion velocity ( $V \exp )$ of the ionized gas in NGC 6565 was derived from the analysis of the cspl in the different ions. Note that the cspl is the same in all the frames, the spectra being radially arranged. Thus, in order to achieve the faintest emissions (like $\lambda 5198-5200 \AA$ of [NI], $\lambda 5517-5537 \AA$ of [ClIII] and $\lambda 7005 \AA$ of $[\mathrm{ArV}])$, the six echellograms were combined.

The results are contained in Table 1, where the ions are put in order of increasing ionization potential (I.P.). Besides the peak separation $(2 V \exp )$ of the main nebula (Col. 4), in Col. 5 we give $V \exp$ of the faint, fast, blueshifted component emitted by the approaching, southern polar cup, clearly visible in Figs. 3 and 4.
As the cspl refers to the kinematical properties of the (almost) polar gas in NGC 6565, so the zvpc contains the spatial properties of the equatorial matter. The intensity peak separations, $2 r_{\mathrm{zvpc}}$, in the different emissions are presented in Cols. 6 to 11 of Table 1.

Typical errors for $2 V \exp \left(\right.$ main) and $2 r_{\mathrm{zvpc}}$ are $1.0 \mathrm{~km} \mathrm{~s}^{-1}$ and 0.15 arcsec for the strongest forbidden emissions ([OI], [SII], [NII] and [OIII]) to $3.0 \mathrm{~km} \mathrm{~s}^{-1}$ and 0.3 arcsec for the faintest ones ([NI], [OII], [ClIII] and $[\mathrm{ArV}])$. The corresponding inaccuracies for the recombination lines are: $2.0 \mathrm{~km} \mathrm{~s}^{-1}$ and $0.2 \operatorname{arcsec}$ for HI, $1.5 \mathrm{~km} \mathrm{~s}^{-1}$ and 0.2 arcsec for HeI and $3.0 \mathrm{~km} \mathrm{~s}^{-1}$ and 0.3 arcsec for HeII. The error in $V \exp (\operatorname{cup})$ (Col. 5 ) is $3.0 \mathrm{~km} \mathrm{~s}^{-1}$ in all cases.

The correlation between cspl and zvpc, evident in Table 1, is summarized in Fig. 6, showing both $V \exp$ and 


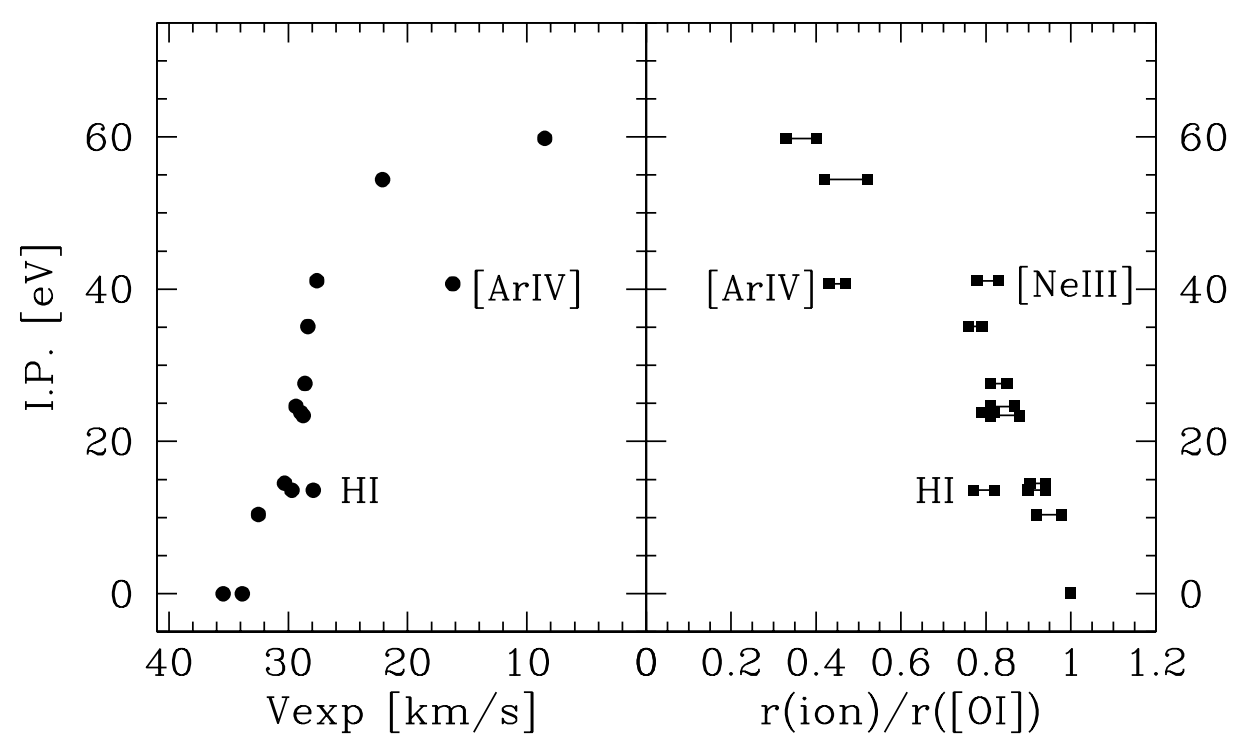

Fig. 6. Polar expansion velocity (left) and equatorial peak separation (right) vs. I.P. in NGC 6565, showing the linear relation between $V \exp$ and distance from the central star. The intervals of the peak separations at different PA, normalized to the [OI] values, are reported. The ions deviating from the normal (cf. text) behavior are marked.

$r_{\mathrm{zvpc}}$ vs. I.P. For reasons of homogeneity, the peak separations at different $\mathrm{PA}$ are normalized to the $[\mathrm{OI}]$ value.

NGC 6565 follows the Wilson's law ("the highexcitation particles show smaller separations than the low excitation particles", Wilson 1950) with some caveats:

- the "high ionization" zone (I.P. > $50 \mathrm{eV}$ ) is poorly defined, containing only two emissions: $\lambda 7005 \AA$ of $[\mathrm{ArV}]$ is exceedingly faint and $\lambda 4686 \AA$ of HeII consists of thirteen fine structure components whose spectral distribution presents a noticeable blue-shifted tail lowering the measurement reliability (in fact, $2 V \exp (\mathrm{HeII})$ from $\lambda 6560 \AA$ is $38 \pm 3 \mathrm{~km} \mathrm{~s}^{-1}$ );

- the "medium ionization" region $(20 \mathrm{eV}<$ I.P. $<50 \mathrm{eV})$ is characterized by a steep velocity gradient. $V \exp$ and $r_{\mathrm{zvpc}}$ in $[\mathrm{ArIV}]$ are considerably lower than in the other ions, as found by Wilson (1950) for NGC 3242 and NGC 7662. On the contrary, [NeIII], a "medium-high" ionization species, is emitted in the "medium-low" excitation region, as observed in NGC 6818, NGC 6886 and NGC 7662 by Wilson (1950), and in NGC 6720 by Reay \& Worswick (1977). These peculiarities will be analyzed in Sect. 9 (photo-ionization model);

- the "low ionization" zone (I.P. $<20 \mathrm{eV}$ ) is steep and well defined, with the obvious exception of hydrogen, which is emitted by the whole ionized nebula, independently on the plasma conditions.

Assuming a minimum degree of symmetry within the nebula we can assess that the expansion velocity is proportional to the distance from the central star, in qualitative agreement with the results of the classical papers of Wilson (1950) and Weedman (1968), performed on a consistent sample of objects.

The exact value of the proportionality constant, $A=$ $V \exp / r^{\prime \prime}$, remains unknown, since at the moment no point of the nebula possesses both the expansion velocity and the distance from the star. Although a rigorous treatment of the problem is presented in Sect. 7, an intuitive solution is evident in Fig. 4: by chance the combination of spatial scale and spectral dispersion in the NTT+EMMI echellograms gives emission line structures of NGC 6565 reproducing the tomographic maps in the slice covered by the slit.

Let's come back to the gas kinematics. Further information is in the position-velocity maps, i.e. in the complete radial velocity field. The maps, shown in Fig. 7 for the six PA, are in the nebula rest frame. The systemic heliocentric velocity is $V r_{\odot}=-5.2 \pm 0.5 \mathrm{~km} \mathrm{~s}^{-1}$. We have selected HeII, [OIII] and [NII] as representative of high, medium and low ionization regions, respectively, and $\mathrm{H} \alpha$, which gives the overall distribution of the ionized gas $\left(\right.$ Flux $(\mathrm{H} \alpha) \propto N \mathrm{e}^{2} \propto N\left(\mathrm{H}^{+}\right)^{2}$, where $N$ e and $N\left(\mathrm{H}^{+}\right)$are the electron and proton densities, respectively).

A number of interesting features can be identified from these maps: the complex expansion velocity field characterized by the polar cups, the fast low excitation blueshifted wisp in $\mathrm{PA}=0^{\circ}$ and $150^{\circ}$, the large stratification effects, the blurred appearance of $\mathrm{H} \alpha$ due to a combination of thermal motions, fine-structure and expansion gradient, and the blue-shifted tail in HeII (asymmetric distribution of the thirteen fine-structure components). In addition, our spectra point out the presence of an external, very faint (untilted?) emission having different kinematical properties with respect to the main nebula. This "halo" (barely visible in Figs. 2, 3 and 7) is more evident in [NII] than in $\mathrm{H} \alpha$ and [OIII]. It appears doublepeaked in [NII] $\left(2 V \exp =25 \pm 3 \mathrm{~km} \mathrm{~s}^{-1}\right)$, with the blueshifted component stronger than the red-shifted one in the southern half of the nebula, and vice-versa in the northern half. In both $\mathrm{H} \alpha$ and [OIII], a single, broad emission $\left(F W H M=25 \pm 5 \mathrm{~km} \mathrm{~s}^{-1}\right)$ is visible. 


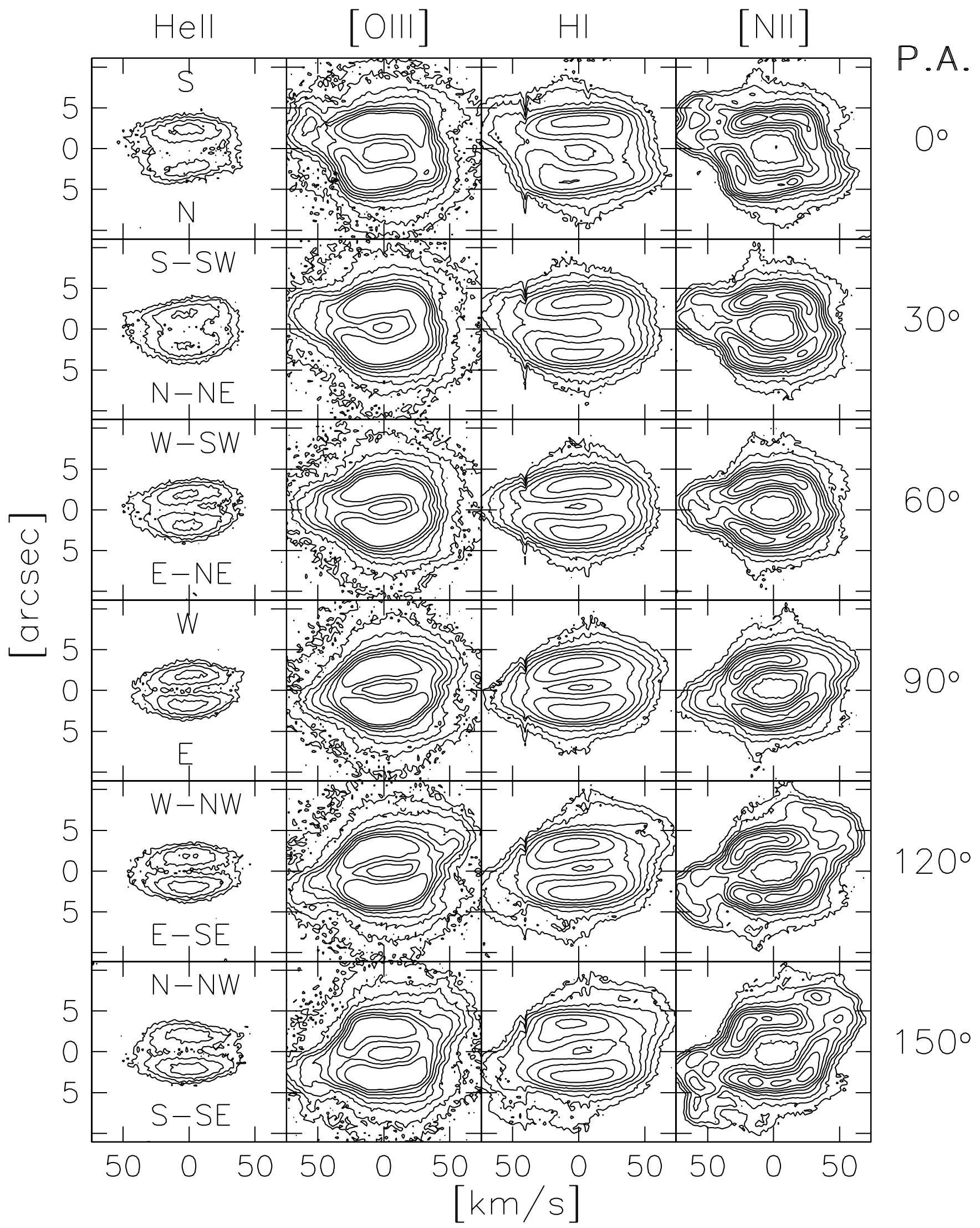

Fig. 7. Position-velocity maps for representative ions of NGC 6565 at the six PA observed. The orientation of each PA is indicated in the HeII panel. The contour step is $\Delta \log F=0.30$. 


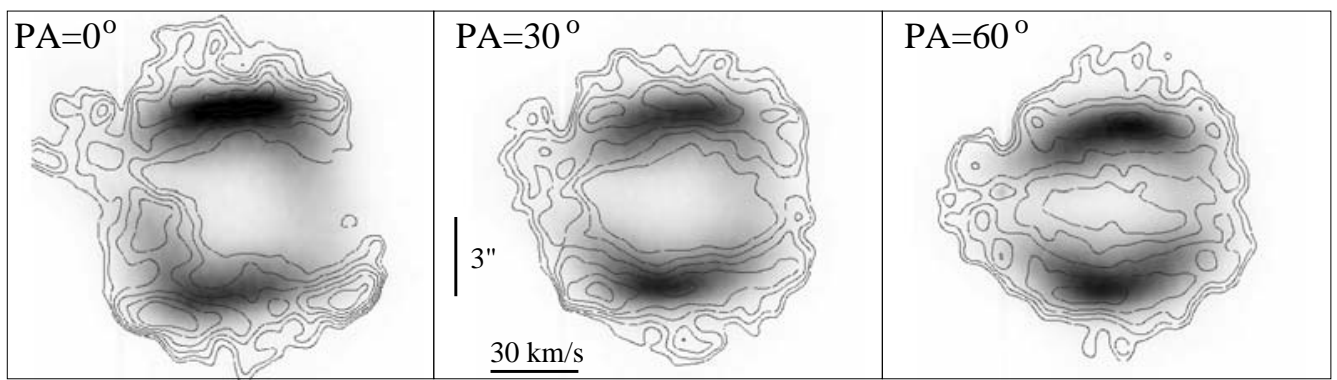

Fig. 8. Isophotal contours of the $\mathrm{H} \alpha / \mathrm{H} \beta$ ratio superimposed to the $\mathrm{H} \alpha$ spectral image for some representative PA of NGC 6565. The orientation is as in Fig. 7. The isophotal contours range between 3.6 (the outermost) and 4.6, with a constant step of 0.2 .

The existence of a faint, low ionization envelope around NGC 6565 supports the Tylenda's $(1983,1986)$ suggestion that the nebula is in a recombination phase.

The only kinematical study of NGC 6565 reported in the literature is by Meatheringham et al. (1988), who derived $2 V \exp =41.8 \mathrm{~km} \mathrm{~s}^{-1}$ in [OII], $28.8 \mathrm{~km} \mathrm{~s}^{-1}$ in [OIII] and $16.6 \mathrm{~km} \mathrm{~s}^{-1}$ in HeII from the peak separation, and $2 V \exp [\mathrm{OIII}]=54.4 \mathrm{~km} \mathrm{~s}^{-1}$ from the width at $10 \%$ maximum intensity (following Dopita et al. 1985, this gives to a good approximation the highest expansion velocity).

The disagreement with the results of Table 1 can be ascribed to the different spatial and spectral accuracies (Meatheringham et al. 1988 used a slit width of 3.9" and a spectral resolution $\simeq 26000$ ), and to the different reduction techniques. To be noticed that the width at $10 \%$ maximum intensity in our echellograms gives $2 \mathrm{~V} \exp [\mathrm{OIII}]=$ $90( \pm 4) \mathrm{km} \mathrm{s}^{-1}$. Further comments will be contained in Sect. 10.2.

\section{The $\mathrm{H} \alpha / \mathrm{H} \beta$ spectral maps}

In general (see Aller 1984; Pottasch 1984; Osterbrock 1989), the PN extinction is obtained by comparing the observed Balmer decrement (in particular $\mathrm{H} \alpha / \mathrm{H} \beta$ ) to the intrinsic value given by Brocklehurst (1971) and Hummer \& Storey (1987). The estimates of $(\mathrm{H} \alpha / \mathrm{H} \beta)_{\mathrm{obs}}$ reported in the literature for NGC 6565 span the range 3.79 (de Freitas Pacheco et al. 1992) to 4.65 (Aller et al. 1988). They all represent mean values over the slice of nebula covered by the slit.

The spatial and spectral accuracies achieved by the superposition technique used $( \pm 0.15$ arcsec and $\pm 1.0 \mathrm{~km} \mathrm{~s}^{-1}$, respectively) allow us to extend the $\mathrm{H} \alpha / \mathrm{H} \beta$ analysis to the whole spectral image. Note that the fine structure of the lines (the spectral distribution and the relative intensities of the seven components of $\mathrm{H} \alpha$ differ from the corresponding quantities of the as many components of $\mathrm{H} \beta$, Clegg et al. 1999) is uninfluent for an expanding gas at $T \mathrm{e} \simeq 10^{4} \mathrm{~K}$.

Figure 8 shows the $\mathrm{H} \alpha / \mathrm{H} \beta$ isophotal contours superimposed to the $\mathrm{H} \alpha$ spectral emission (recall that $\operatorname{Flux}(\mathrm{H} \alpha) \propto$ $\left.N\left(\mathrm{H}^{+}\right)^{2}\right)$ for some representative PA of NGC 6565. The large scale appearance of the Balmer ratio is the same in all the six spectra: there is a minimum $(3.6-3.8)$ in the central regions, it increases outwards (up to $4.4-4.6$ just beyond the $\mathrm{H} \alpha$ peaks), then gradually decreases to 3.7-4.1 (the last statement is uncertain, because of the low signalto-noise in the outer regions).

So far, $\mathrm{H} \alpha / \mathrm{H} \beta$ variations were reported for a few $\mathrm{PNe}$ by comparing direct, narrow-band imagery, and ascribed to the presence of absorbing dust within (or around) the objects (for example, in NGC 6302 by Bohigas 1994, in NGC 6445 by Cuesta \& Phillips 1999, in NGC 2440 by Cuesta \& Phillips 2000, and in NGC 6781 by Mavromatakis et al. 2001).

The advantage of a "spectral map" is evident: it shows the de-projected slice of nebula covered by the slit, whereas the "direct images superposition" refers to the PN projection on the sky.

In general, two main factors affect the $\mathrm{H} \alpha / \mathrm{H} \beta$ ratio:

a) the amount of dust along the line of sight. We can write:

$$
c(\mathrm{H} \beta)_{\text {tot }}=c(\mathrm{H} \beta)_{\text {interstellar }}+c(\mathrm{H} \beta)_{\text {local }}
$$

where $\mathrm{c}(\mathrm{H} \beta)_{\text {local }}$ is null for optically thin, density bounded PNe. But NGC 6565 is decidedly optically thick to the UV stellar radiation, mainly in the dense, equatorial regions. Moreover, Gathier et al. (1986) and Stasinska et al. (1992) suggest that the infrared and radio excesses of the nebula could be due to a large amount of local absorbing material. Our $\mathrm{H} \alpha / \mathrm{H} \beta$ spectral maps indicate that the neutral gas, if present, mainly surrounds the equatorial regions;

b) the $T$ e distribution in the ionized gas: the higher the electron temperature, the smaller the intrinsic $\mathrm{H} \alpha / \mathrm{H} \beta$ ratio. For example, we have $\mathrm{H} \alpha / \mathrm{H} \beta=2.74,2.85$ and 3.02 for $T \mathrm{e}=20000 \mathrm{~K}, 10000 \mathrm{~K}$ and $5000 \mathrm{~K}$, respectively (assuming the case B of Baker \& Menzel 1938, and $\log \mathrm{Ne}=3.00$; Brocklehurst 1971; Aller 1984; Hummer \& Storey 1987).

A radial variation of $T$ e within NGC 6565 is expected, due to the complexity of the ionization structure.

Note that a large amount of dust in the equatorial regions of the nebula should produce spatial and wavelengthasymmetric $\mathrm{H} \alpha / \mathrm{H} \beta$ spectral maps, whereas a radial Te variation should not. Unfortunately, our resolutions are inadequate (by a factor of almost 2) to test such a possibility. In the present case, the discrimination between a) 


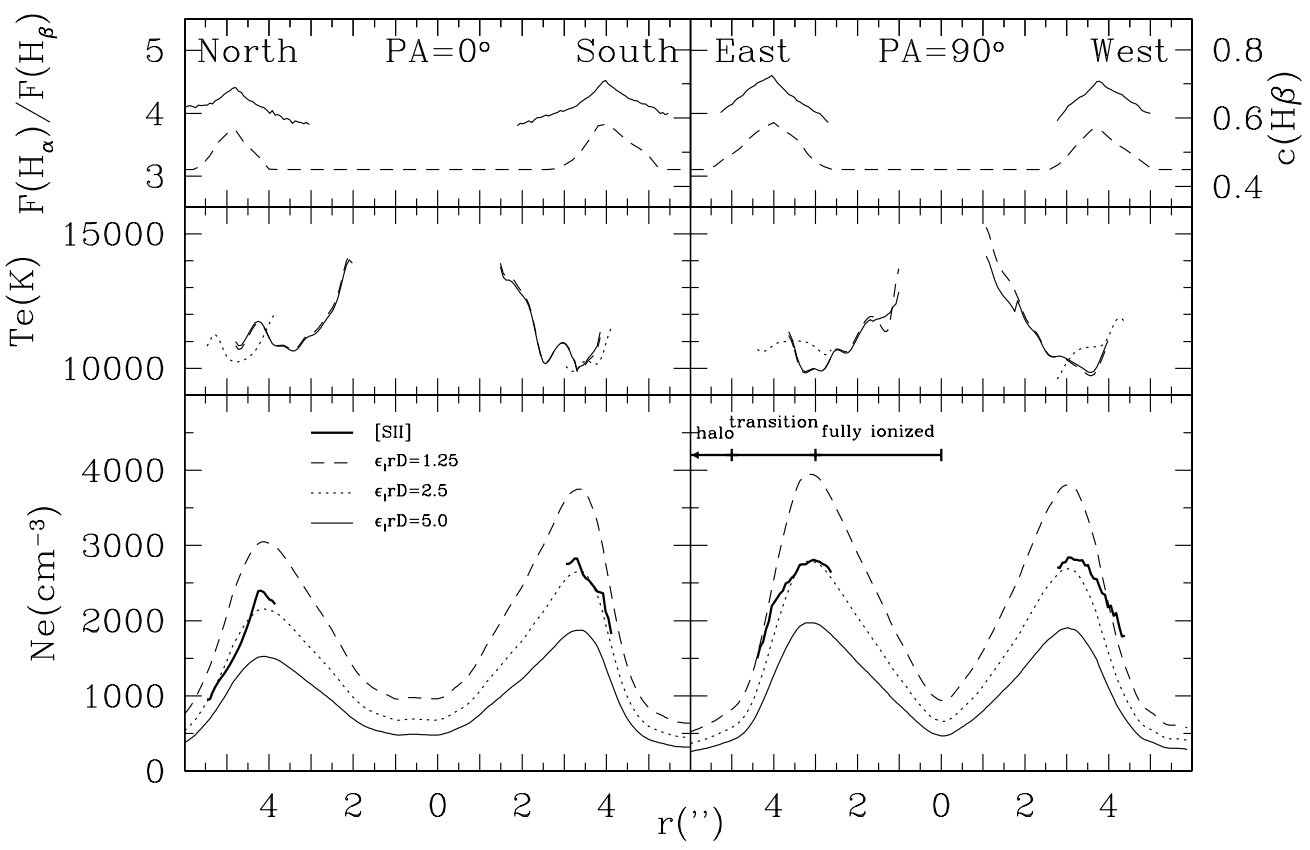

Fig. 9. Radial physical conditions and other parameters in NGC 6565 at $\mathrm{PA}=0^{\circ}$ (left) and $90^{\circ}$ (right). Top panel: the observed $\mathrm{H} \alpha / \mathrm{H} \beta$ profile in the zvpc (left ordinate scale; continuous line) and the adopted $c(\mathrm{H} \beta)_{\text {tot }}$ profile (right ordinate scale; dashed line). Middle panel: $T \mathrm{e}[\mathrm{OIII}]$ for cases A (i.e. $c(\mathrm{H} \beta)$ variable across the nebula; continuous line) and $\mathrm{B}(c(\mathrm{H} \beta)=$ constant $=0.45$; long-dashed line) and $T \mathrm{e}[\mathrm{NII}]$ (dotted line). Bottom panel: $N \mathrm{e}[\mathrm{SII}]$ (thick continuous line) and $N$ e from the H $\alpha$ flux for some representative values of $\epsilon_{1} \times r_{\mathrm{cspl}} \times D(\operatorname{arcsec} \mathrm{kpc})$. The three radial zones discussed in the text are indicated in the lower right panel.

and b) requires a detailed analysis of the nebular physical conditions.

\section{The physical conditions}

The radial profiles of $T \mathrm{e}$ and $\mathrm{Ne}$ are obtained from the classical diagnostic line ratios (ions in $\mathrm{p}^{3}$ configuration for $\mathrm{Ne}$ and in $\mathrm{p}^{2}$ or $\mathrm{p}^{4}$ configurations for Te; Aller 1984; Osterbrock 1989) and from the absolute $\mathrm{H} \alpha$ flux distribution (Sabbadin et al. 2000a,b; Ragazzoni et al. 2001). A compilation of the relevant references for the transition probabilities and collision strengths used in this paper to derive $T$ e, $N$ e and the ionic and chemical composition is given by Hyung \& Aller (1996) and Liu et al. (2000).

In all cases we make use of the zvpc, which is independent on the expansion velocity field, and corresponds to the equatorial, densest nebular regions of NGC 6565. The logical steps of our analysis are the following:

- first we obtain Te[OIII] from $\lambda 5007 \AA / \lambda 4363 \AA$ (the line ratio is almost independent on $N$ e for $N$ e $\left.<10^{4} \mathrm{~cm}^{-3}\right)$;

- $N$ e[SII] is then derived from $\lambda 6717 \AA / \lambda 6731 \AA$ (using $T$ e[OIII] to take into account the weak dependence of the ratio on $T \mathrm{e})$;

- $T$ e[NII] comes from $\lambda 6584 \AA / \lambda 5755 \AA$ (adopting $N$ e[SII] for its weak dependence on $\mathrm{Ne}$ );

- last, $\mathrm{Ne}(\mathrm{H} \alpha)$ is given by the $\mathrm{H} \alpha$ flux distribution in the zvcp (both $T \mathrm{e}[\mathrm{OIII}]$ and $T \mathrm{e}[\mathrm{NII}]$ are considered for the $T$ e dependence of the $\mathrm{H} \alpha$ emissivity).

The other diagnostics present in the spectra (like $\lambda 4711 \AA / \lambda 4740 \AA$ of $[\mathrm{ArIV}], \lambda 5198 \AA / \lambda 5200 \AA$ of $[\mathrm{NI}]$, $\lambda 5517 \AA / \lambda 5537 \AA$ of $[\mathrm{ClIII}]$ and the $[\mathrm{OII}]$ red quartet around $\lambda 7325 \AA$ ) are too weak for an accurate spatial analysis.

This procedure, performed at all the six PA of NGC 6565, gives very similar results, as expected for an ellipsoid seen almost pole-on. Thus, only two representative $\mathrm{PA}$ are illustrated here: $\mathrm{PA}=0^{\circ}$ (along the apparent major axis) and $\mathrm{PA}=90^{\circ}$ (along the apparent minor axis). The results are summarized in Fig. 9.

\section{1. $\mathrm{Te}[\mathrm{OIII}]$}

In order to get the intrinsic line ratio $I(\lambda 5007 \AA) /$ $I(\lambda 4363 \AA)$, the observed line intensities must be dereddened. We have just found that $\mathrm{H} \alpha / \mathrm{H} \beta$ systematically changes over NGC 6565 and, at the moment, we cannot identify the main cause for the variation ( $T$ e or absorption). Thus, we will consider two extreme cases:

A) the $\mathrm{H} \alpha / \mathrm{H} \beta$ ratio only depends on the amount of absorption along the line of sight. In practice this corresponds to assume a constant $T \mathrm{e}=10000 \mathrm{~K}$;

B) the absorption is constant over the nebula and the $\mathrm{H} \alpha / \mathrm{H} \beta$ variation is due to $T$ e. We discuss the case of $c(\mathrm{H} \beta)=0.45$; the choice of other, reasonable values of $c(\mathrm{H} \beta)$ doesn't modify the conclusions here obtained.

The resulting $T$ e[OIII] profiles, shown in Fig. 9, middle panel, appear very similar in the two cases: the electron temperature is large $(14000-15000 \mathrm{~K})$ in the innermost regions, it rapidly decreases outwards down to about 
$10000 \mathrm{~K}$, and then it slightly increases (the last statement is weakened by the faintness of the [OIII] auroral emission). Previous Te[OIII] determinations in NGC 6565 are mean values and range from $9700 \mathrm{~K}$ (Acker et al. 1991) to $10300 \mathrm{~K}$ (Aller et al. 1988; de Freitas Pacheco et al. 1992).

The knowledge of the detailed $T \mathrm{e}[\mathrm{OIII}]$ radial profile allows us to eliminate the dependence of the $\mathrm{H} \alpha / \mathrm{H} \beta$ ratio on $T$ e, thus obtaining $\mathrm{c}(\mathrm{H} \beta)$. Our results indicate that:

- $c(\mathrm{H} \beta)_{\text {interstellar }}=0.45( \pm 0.02)$;

- in the innermost regions $c(\mathrm{H} \beta)_{\text {local }} \simeq 0$. Once corrected for the interstellar component, the ratio $\mathrm{H} \alpha / \mathrm{H} \beta$ is low, due to the large $T$ e of the ionized gas. Te decreases outwards and, accordingly, $\mathrm{H} \alpha / \mathrm{H} \beta$ increases;

- the $\mathrm{H} \alpha / \mathrm{H} \beta$ rise beyond the $\mathrm{H} \alpha$ intensity peak is mainly caused by the presence of neutral absorbing material around the ionized nebula. The maximum of $c(\mathrm{H} \beta)_{\text {local }}$, $0.12( \pm 0.03)$, occurs at about $1.2( \pm 0.1) \times r_{\mathrm{H}^{+}}\left(r_{\mathrm{H}^{+}}\right.$ being the radius of the $\mathrm{H} \alpha$ peak).

The adopted, final $c(\mathrm{H} \beta)_{\text {tot }}$ profile for the two selected PA of NGC 6565 is shown in Fig. 9, top panel. It will be used in the next sections to correct the observed intensities through the relation:

$\log \frac{I(\lambda)_{\text {corr }}}{I(\lambda)_{\mathrm{obs}}}=f_{\lambda} c(\mathrm{H} \beta)$

where $f_{\lambda}$ is the interstellar extinction coefficient given by Seaton (1979).

Notice that the adopted $c(\mathrm{H} \beta)_{\text {tot }}$ profile is obtained under the assumption $T \mathrm{e}\left(\mathrm{H}^{+}\right) \simeq T \mathrm{e}[\mathrm{OIII}] \simeq T \mathrm{e}[\mathrm{NII}]$; this is questionable in the outermost (knotty) regions, where the ionization drops and $\mathrm{H}^{+}$and $\mathrm{H}^{0}$ co-exist.

Lowering $T$ e(almost neutral gas), $c(\mathrm{H} \beta)_{\text {local }}$ decreases and goes to zero for $T$ e(almost neutral gas $) \simeq 5000 \mathrm{~K}$. In this case the overall $\mathrm{H} \alpha / \mathrm{H} \beta$ spectral distribution of Sect. 5 is due to $T$ e variations. On the one hand this modifies only moderately the de-reddened line fluxes, on the other hand it implies that $\mathrm{H}^{+} \gg \mathrm{H}^{\circ}$ (in other words: if the electron temperature of the almost neutral gas around NGC 6565 is $\simeq 5000 \mathrm{~K}$, there is no neutral gas!). We will deep the question in the next sections.

\section{2. $\mathrm{Ne}[\mathrm{SII}]$}

The intensity ratio $\lambda 6717 \AA / \lambda 6731 \AA$ of [SII] depends strongly on $N$ e and weakly on $T$ e (Aller 1984; Osterbrock 1989). The accurate knowledge of the $c(\mathrm{H} \beta)$ profile is unimportant, because the two lines are very close. The resulting $N$ e[SII] distribution for the two selected PA of NGC 6565 is included in Fig. 9, bottom panel (thick continuous line). Our analysis suggests that:

- the $N$ e[SII] profile is aligned to the $\mathrm{H} \alpha$ one, and both peak internally to the $\lambda 6717 \AA$ and $\lambda 6731 \AA$ maxima (which, in their turn, are lightly misaligned). This indicates that the $\mathrm{S}^{+}$emission occurs in a recombining region;
- the $N$ e[SII] distribution shows peaks up to $2800 \pm$ $200 \mathrm{~cm}^{-3}$;

- the [SII] density peak is anticorrelated to $r_{\mathrm{zvpc}}$;

- the $\mathrm{S}^{+}$emitting zone, being co-spatial to the $\mathrm{N}^{+}$one (see Fig. 1), has a patchy and inhomogeneous structure favoring the detection of the densest, i.e. brightest, parts and indicating that the local filling factor, $\epsilon_{1}$, in the external, low ionization layers of NGC 6565 is $\epsilon_{1}<1$.

Previous $N$ e[SII] determinations in the nebula (mean values) date from Aller et al. $\left(1988, N e=1600 \mathrm{~cm}^{-3}\right)$, Acker et al. (1991, $\left.\mathrm{Ne}=1460 \mathrm{~cm}^{-3}\right)$ and de Freitas Pacheco et al. (1992, $\left.\mathrm{Ne}=1000 \mathrm{~cm}^{-3}\right)$. Moreover, Meatheringham et al. (1988) obtain $N \mathrm{e}=1550 \mathrm{~cm}^{-3}$ from $\lambda 3726 \AA / \lambda 3729 \AA$ of $[\mathrm{OII}]$.

\section{3. $\mathrm{Te}[\mathrm{NII}]$}

The line intensity ratio $\lambda 6584 \AA / \lambda 5755 \AA$ of $[\mathrm{NII}]$ depends strongly on $T$ e and weakly on $N$ e (Aller 1984; Osterbrock 1989). Adopting the $\mathrm{Ne}[\mathrm{SII}]$ distribution we have derived the Te[NII] one shown in Fig. 9 (middle panel, dotted line). Te[OIII] and $T e[\mathrm{NII}]$ are only partially superimposed: [OIII] is present all over NGC 6565, whereas [NII] is peaked in the outermost, low ionization regions. Their values are similar; the electron temperature discrepancy, normally observed in $\mathrm{PNe}$, can be ascribed to inaccuracy in the atomic parameters, small scale ionization fluctuation and, mainly, weakness of the auroral lines (Aller 1990; Gruenwald \& Viegas 1995; Mathis et al. 1998). Previous Te[NII] determinations in NGC 6565 are mean values and span in the range 8900-9500 K (Aller et al. 1988; Acker et al. 1991; de Freitas Pacheco et al. 1992).

\subsection{Ne from the Ho flux}

Following Sabbadin et al. (2000a,b) and Ragazzoni et al. (2001), the accurate $N$ e radial distribution can be obtained from the absolute $\mathrm{H} \alpha$ flux in the zvpc. The dereddened $\mathrm{H} \alpha$ profile must be further corrected for the instrumental resolution, thermal motions and fine structure (for details, see Sabbadin et al. 2000a). These corrections were superfluous for the diagnostics just analyzed, i.e. $\lambda 5007 \AA / \lambda 4363 \AA, \lambda 6717 \AA / \lambda 6731 \AA$ and $\lambda 6584 \AA / \lambda 5755 \AA$, because they are forbidden lines and each ratio refers to the same ion and is emitted in the same nebular region.

For a pixel of the $\mathrm{H} \alpha$ zvpc we have:

$4 \pi D^{2} F(\mathrm{H} \alpha)=4 \pi j_{\mathrm{H} \alpha} N\left(\mathrm{H}^{+}\right) N e V_{\mathrm{l}} \epsilon_{\mathrm{l}}$

where:

- $D$ is the nebular distance;

- $j_{\mathrm{H} \alpha}$ is the emission coefficient (case B of Baker \& Menzel 1938), interpolated from Brocklehurst (1971) values;

- $V_{1}$ is the "local volume", i.e. the nebular volume identified by a single pixel of the zvpc; $V_{\mathrm{l}}$ is given by $l \times b \times s \times(D / 206265)^{3}$, where $l=\operatorname{slit}$ width $\left(1.0^{\prime \prime}\right)$, 
$b=$ pixel spatial scale $\left(0.27^{\prime \prime}\right)$ and $s$ is the radial depth of the zvpc, $s=r_{\mathrm{cspl}} \Delta V / V \exp$, with $\Delta V=$ pixel spectral resolution $\left(1.79 \mathrm{~km} \mathrm{~s}^{-1}\right.$ ), $V \exp =28 \mathrm{~km} \mathrm{~s}^{-1}$ (see Table 1) and $r_{\text {cspl }}$ is the (still unknown) angular radius of the cspl (i.e. the $\mathrm{H} \alpha$ nebular size in the radial direction);

$-\epsilon_{1}$, the "local filling factor", corresponds to the fraction of the local volume $V_{1}$ which is actually filled by matter with density $N$ e.

We can make the reasonable assumptions that $N \mathrm{e}=1.15 \times$ $N\left(\mathrm{H}^{+}\right)$and $\epsilon_{1}=$ constant along the zvpc, thus obtaining:

$N \mathrm{e}=\frac{1.07 \times 10^{9}}{T \mathrm{e}^{-0.47}} \times\left(\frac{F(\mathrm{H} \alpha)}{\epsilon_{\mathrm{l}} \times r_{\mathrm{cspl}} \times D}\right)^{1 / 2}$.

Equation (4), combined with $N e \simeq N e[S I I]$, represents an important link between the spatial and the dynamical properties of the ionized gas (i.e. between the zvpc and the cspl). It can be used to derive the distance if independent information on the radial radius, $r_{\mathrm{cspl}}$, is available (for example, $\epsilon_{1}=1$ and $r_{\mathrm{cspl}}=r_{\mathrm{zvpc}}$ in a homogeneous $\mathrm{PN}$ presenting a large degree of symmetry). In the case of NGC 6565 we have the opposite situation: the distance will be obtained in the next section by means of the extinctiondistance correlation; thus, Eq. (4) will be used to derive $\epsilon_{1}$ and the radial size of the cspl.

Figure 9, bottom panel, shows the $N$ e distribution for three representative values of $\epsilon_{1} \times r_{\mathrm{cspl}} \times D(1.25,2.5$ and 5.0 arcsec kpc). Note the $N$ e radial asymmetry (the decline outward the maximum is steeper than the internal rise), and the similarity with the [SII] density profile, the alignment of the peaks and the close overlap for $\epsilon_{1} \times r_{\text {cspl }} \times D=2.5$ arcsec kpc.

\subsection{Physical conditions: general considerations}

Our analysis shows that the equatorial regions of NGC 6565 consist of three, distinct radial zones, indicated in the right-bottom panel of Fig. 9:

- the "fully ionized" nebula is the innermost one (roughly extending up to the $\mathrm{H} \alpha$ peak). Ne monotonically increases outwards, while $T$ e first decreases then remains almost constant;

- the "transition" zone located just beyond the electron density peak. Here $N$ e rapidly decreases to a few hundreds $\mathrm{cm}^{-3}$ and $T$ e softly increases outwards;

- the outermost nebula, the "halo", presenting a gentle gradient of $N e$; nothing can be said on $T$ ehalo $_{\text {. }}$.

Note that:

- the overall $N$ e profile can be obtained from the $\mathrm{H} \alpha$ flux in the zvpc, whereas $T$ e is limited to the main nebula, due to the weakness of the [OIII] and [NII] auroral lines. Much deeper echellograms are necessary (and highly advisable) to determine $T$ e up to the faintest regions;

- the $N$ e of the halo shown in Fig. 9 (bottom panel) must be considered as an upper limit since we have adopted the same kinematical properties for the main nebula and the halo. A better approximation is given by:

$$
N \mathrm{e}_{\text {halo }}=\frac{N \mathrm{e}_{\text {halo }}(\text { Fig. 9) }}{\left[\frac{r_{\text {halo }}}{r_{\text {main }}} \times \frac{V \exp _{\text {main }}}{V \exp _{\text {halo }}}\right]^{1 / 2}} \simeq \frac{N e_{\text {halo }}(\text { Fig. 9) }}{2}
$$

- Te refers to the ionized gas, but there are growing evidences indicating the presence of a considerable amount of almost neutral, patchy gas (at unknown $T$ e) in both the "transition" zone and the "halo".

Up to now we have obtained the physical conditions in the equatorial regions of NGC 6565 using the zvpc. The linear relation between expansion velocity and nebular radius, found in Sect. 4, suggests that the same analysis can be performed also in the polar regions. The main problem is represented by the line fluxes, which are considerably lower in the cspl than in the zvpc.

Only a rough $N$ e profile can be derived for the (almost) polar regions, showing density peaks of $1000( \pm 200) \mathrm{cm}^{-3}$ in $[\mathrm{SII}]$ and $500( \pm 100) \mathrm{cm}^{-3}$ from the $\mathrm{H} \alpha$ flux (adopting $T \mathrm{e}=10^{4} \mathrm{~K}$ and $\epsilon_{\mathrm{l}}=1$; see Sect. 7.2).

\section{The nebular distance, radius and mass and the central star parameters}

\subsection{Distance}

In Sect. 5 we have obtained $c(\mathrm{H} \beta)_{\text {interstellar }}=0.45 \pm 0.02$, corresponding to $E(B-V)=0.31 \pm 0.01$ (Acker 1978). The introduction of this value in the reddening-distance diagrams presented by Gathier et al. (1986, weight 2), Maciel et al. (1986, weight 1) and de Oliveira-Abans \& Faundez-Abans (1991, weight 1) gives a nebular distance of $2.0 \pm 0.5 \mathrm{kpc}$. Previous individual estimates, based on lower values of $c(\mathrm{H} \beta)$, span the range 0.5 to $1.55 \mathrm{kpc}$, whereas statistical distances are spread between 0.9 (Gathier 1987) and $7.7 \mathrm{kpc}$ (Kingsburgh \& Barlow 1992).

Using the scheme introduced in the previous section and $D=2.0 \mathrm{kpc}$ we have:

$R_{\text {fully ionized }}=0.035 \times 0.029 \mathrm{pc}$

$R_{\text {transition }}=0.054 \times 0.048 \mathrm{pc}$

$R_{\text {halo }} \simeq 0.11$ pc (indicative value).

\subsection{Filling factor and $r_{\text {cspl }}$}

Recalling, from sub-section 6.4, that the best match of $N \mathrm{e}[\mathrm{SII}]$ with $N \mathrm{Ne}(\mathrm{H} \alpha)$ occurs for $\epsilon_{1} \times r_{\text {cspl }} \times D=$ 2.5 arcsec $\mathrm{kpc}$, we immediately have $\epsilon_{1} \times r_{\mathrm{cspl}}=$ 1.25 arcsec. Note that for $\epsilon_{1} \sim 1$ we obtain ellipsoids compressed along the polar axis $\left(r_{\mathrm{cspl}}=1.25\right.$ arcsec, i.e. $r$ (equatorial $) / r$ (polar $) \simeq 2.6)$. Equally improbable are the solutions for $1 \gg \epsilon_{1}$; for $\epsilon_{1}=0.1$ we have $r_{\mathrm{cspl}}=$ 12.5 arcsec, that is a nebula very stretched along the polar axis; $r$ (equatorial) $/ r$ (polar $) \simeq 0.26$.

A more stringent upper limit to $\epsilon_{1}, \epsilon_{1} \leq 0.35$, results from the intuitive assumption $r$ (polar) $\geq r$ (equatorial), 
i.e. $r_{\mathrm{cspl}} \geq r_{\mathrm{zvpc}}$. Moreover, by extrapolating to the polar regions the anticorrelation between $N \mathrm{Ne}[\mathrm{SII}]$ (peak) and $r_{\mathrm{zvpc}}$ found for the equatorial zone (cf. Sect. 6.2), we derive $r_{\mathrm{cspl}} \simeq 5.0 \operatorname{arcsec}$ (and $\epsilon_{1} \simeq 0.25$ ). This is confirmed by the morphological analysis of the "NGC 6565like" PNe projected more or less equatorial-on, contained in the catalogues of Acker et al. (1992), Schwarz et al. (1992), Manchado et al. (1996), and Gorny et al. (1999), e.g. NGC 6886 (PNG 060.1-07.7; see Fig. 5), M 2-40 (PNG 024.1+03.8), K 3-92 (PNG 130.4+03.1), M 2-53 (PNG 104.4-01.6) and M 1-7 (PNG 189.8+07.7).

Thus, in the following we will adopt $r_{\mathrm{cspl}}=5.0 \pm$ $0.5 \operatorname{arcsec}$ (corresponding to $0.048 \pm 0.005 \mathrm{pc}$ ), and $\epsilon_{1}=0.25$, constant across the $\mathrm{S}^{+}$emitting region (consistent with the patchy structure of the low ionization layers). The position-velocity proportionality constant, $A=$ $V \exp / r_{\text {cspl }}$, results to be $5.6 \pm 0.6 \mathrm{~km} \mathrm{~s}^{-1} \operatorname{arcsec}^{-1}$; this agrees with the impression that the NTT+EMMI emission line structure of NGC 6565 nicely reproduces (to within $20 \%$ ) the tomographic map of the nebular slice covered by the slit; in fact, in Figs. 3, 4, 7 and 8 one arcsec corresponds to 3.11 pixels along the dispersion (cspl) and to 3.70 pixels along the slit (zvpc).

The kinematical age of the nebula, $t_{\text {kin }} \propto A^{-1} \simeq$ $1750 \mathrm{yr}$, represents a lower limit to the actual age, $t_{\mathrm{N} 6565}$, since the dynamical history of the gas is unknown (Aller 1984; Schmidt-Voigt \& Köppen 1987; Dopita et al. 1996). An estimate of $t_{\mathrm{N} 6565}$ can be obtained by assuming a nebular ejection at $V \exp ($ superwind $)<V \exp (N 6565)$, followed by a constant acceleration up to $V \exp (N 6565)$. $V \exp ($ superwind) can be measured in the $\mathrm{OH} / \mathrm{IR}$ sources (i.e. stars surrounded by thick opaque envelopes of gas and dust producing maser emissions), which are believed to be the PNe progenitors (see Habing 1996 and references therein). For $V \exp ($ superwind $)=15( \pm 5) \mathrm{km} \mathrm{s}^{-1}$ (David et al. 1993; Chengalur et al. 1993; Sjouwerman et al. 1998) we derive $t_{N 6565}=2300( \pm 300) \mathrm{yr}$.

\subsection{Nebular mass}

Given the distance, the ionized nebular mass is obtainable in different ways (from the $\mathrm{H} \beta$ flux, the radio flux and the observed Ne distribution; Aller 1984; Pottasch 1984; Osterbrock 1989). Adopting $D=2.0 \mathrm{kpc}$, we get $M_{\text {ion }}=$ $0.03( \pm 0.01) M_{\odot}$, that is considerably lower than the mean nebular mass of optically thin $\mathrm{PNe}, M_{\mathrm{PNe}} \simeq 0.10-0.15 M_{\odot}$ (Pottasch 1983; Boffi \& Stanghellini 1994; Dopita et al. 1996). This confirms that NGC 6565 is ionization bounded and suggests the presence of a considerable amount of surrounding, neutral gas. From simple geometrical considerations we can put $M_{\mathrm{PNe}} / M_{\mathrm{ion}} \simeq\left(R_{\mathrm{tot}} / R_{\mathrm{ion}}\right)^{4}$, where $R_{\mathrm{tot}}$ is the radius of the (ionized + neutral) density peak. We have $R_{\text {tot }} \simeq 1.4 \times R_{\text {ion }}$, i.e. similar to the radius of the peak of $c(\mathrm{H} \beta)$ given by the $\mathrm{H} \alpha / \mathrm{H} \beta$ spectral maps.

An estimate of the neutral gas density, $N\left(\mathrm{H}^{0}\right)$, at $R_{\text {tot }}$ can be obtained from $E(B-V)_{\text {local }}=c(\mathrm{H} \beta)_{\text {local }} / 1.48$ (Acker 1978), taking a "normal" gas to dust ratio
(Spitzer 1978) and assuming a radial thickness of the absorbing layer comparable to $R_{\text {tot }}$. The resulting value, $N\left(\mathrm{H}^{0}\right) \simeq 3( \pm 1) \times 10^{3} \mathrm{~cm}^{-3}$, agrees with all the previous indications that a dense, neutral envelope surrounds the ionized nebula.

A further, independent confirmation comes from the detailed analysis of both the consistency and distribution of the dark globules superimposed on the [OIII] image of NGC 6565 (Fig. 1):

- a number of intensity scans secured in the neighbourhood of the globules indicates a depletion of the [OIII] flux up to $30 \%$, corresponding to $c(\mathrm{H} \beta)_{\text {globules }}$ up to 0.11 (to be compared with $c(\mathrm{H} \beta)_{\text {local }}=0.12 \pm 0.03$ obtained in Sect. 5 from the $\mathrm{H} \alpha / \mathrm{H} \beta$ spectral maps);

- most globules appear in the northern part of the nebula, as expected of an ellipsoid denser in the equatorial regions and seen almost pole-on. Figures 3, 4 and 7 show that the southern polar cup of NGC 6565 is approaching, thus the shadowing by the neutral, dense gas located in the equatorial regions mainly affects the northern portion of the image.

All this, added to the different general morphology of the nebula in [OIII] and [NII] (quite homogeneous and diffuse in [OIII], inhomogeneous and patchy in [NII]; Fig. 1) indicates the presence of noticeable density fluctuations at small spatial scale (below the seeing, $\simeq 0.7$ arcsec) in the outermost, recombining nebular regions, which are rich of knots, globules and condensations.

\subsection{Central star parameters}

The accurate value of $m_{\mathrm{V} *}$ has been obtained from WFPC2 frames of NGC 6565 taken through the broadband filter $F 555 W\left(\lambda_{c}=5407 \AA\right.$, bandwidth $\left.=1236 \AA\right)$. The resulting magnitude is $m_{\mathrm{V} *}=18.88( \pm 0.05)$, where the unknown star color is the main source of inaccuracy. Previous $m_{\mathrm{V} *}$ estimates reported in the literature span the range 15.9 (Shaw \& Kaler 1989) to 20.3 (Gathier \& Pottasch 1988).

The HI and HeII Zanstra temperatures, $T_{\mathrm{Z}} \mathrm{HI}$ and $T_{\mathrm{Z}} \mathrm{HeII}$ respectively, are derived in the classical way (Aller 1984; Pottasch 1984; Osterbrock 1989) by comparing the de-reddened $\mathrm{H} \beta$ and HeII $\lambda 4686 \AA$ nebular fluxes with $\left(m_{\mathrm{V} *}\right)_{\mathrm{o}}$. The integrated $\mathrm{H} \beta$ and $\lambda 4686 \AA$ fluxes were extrapolated from the overall line profile at each PA, assuming a circular symmetry of the nebular image. We obtain $\log F(\mathrm{H} \beta)_{\mathrm{obs}}=-11.20( \pm 0.03) \mathrm{mW} \times \mathrm{m}^{-2}$ and $I(\lambda 4686 \AA / \mathrm{H} \beta)_{\mathrm{obs}}=0.12( \pm 0.02)$. The corresponding values reported in the literature are in the range -11.27 (photoelectric photometry; O'Dell 1962) to -11.1 (slit spectroscopy; Acker et al. 1991) for $\log F(\mathrm{H} \beta)_{\text {obs }}$, and 0.12 (photoelectric narrow-band photometry; Kohoutek \& Martin 1981) to 0.22 (slit spectroscopy; Acker et al. 1991) for $I(\lambda 4686 \AA / \mathrm{H} \beta)_{\text {obs }}$.

We derive $\log \left(T_{\mathrm{Z}} \mathrm{HI}\right)=5.20( \pm 0.05)$ and $\log \left(T_{\mathrm{Z}} \mathrm{HeII}\right)=5.08( \pm 0.05)$, thus confirming the peculiarity already reported by Pottasch (1981), 


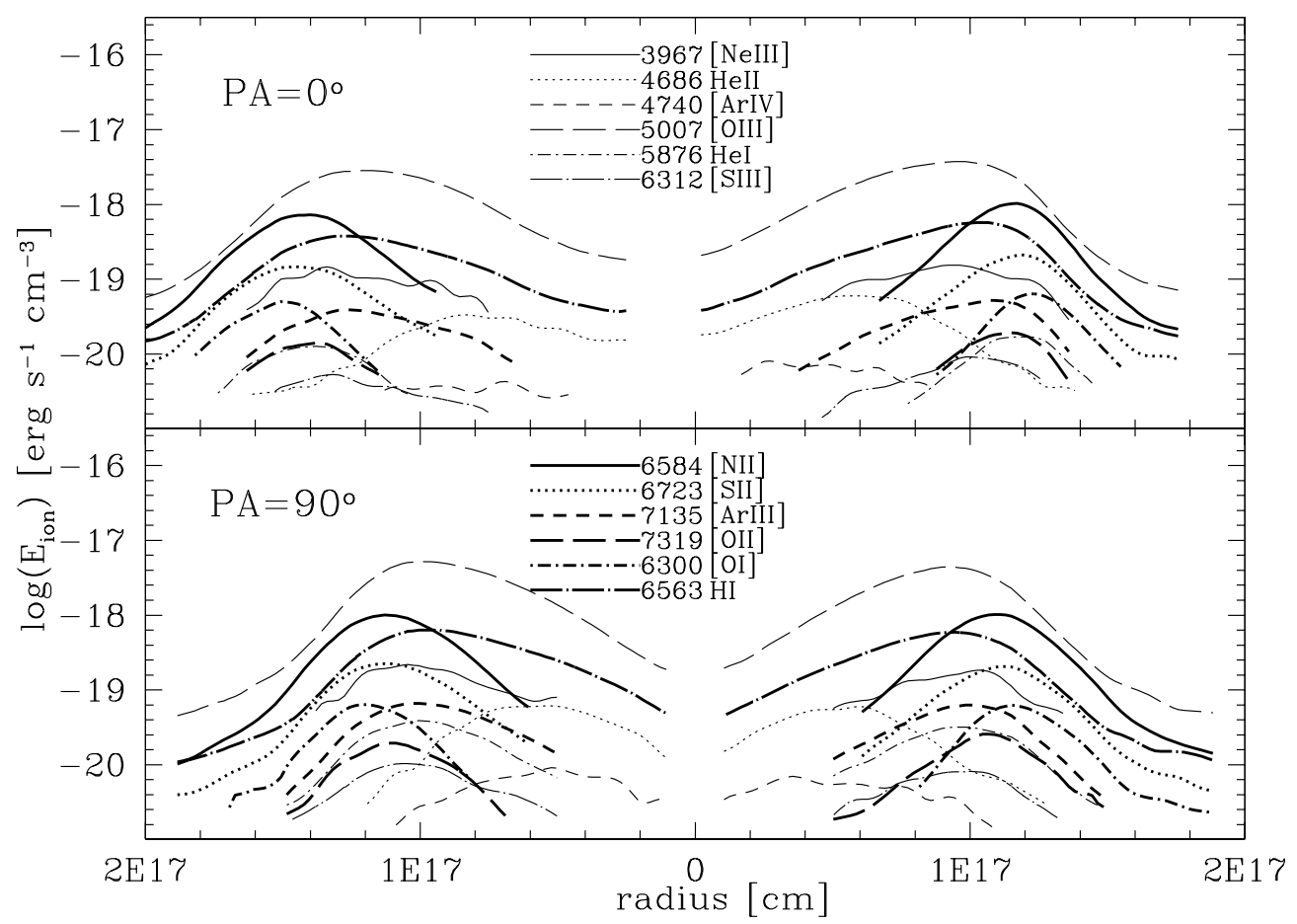

Fig. 10. Absolute emission line radial profiles $\left(\mathrm{erg} \mathrm{cm} \mathrm{cm}^{-3} \mathrm{~s}^{-1}\right.$; logarithmic scale) in the zvpc of NGC 6565 at $\mathrm{PA}=0^{\circ}$ and $90^{\circ}$. The orientation is as in Fig. 9. The nebula has been considered at a distance $D=2.0 \mathrm{kpc}$.

Martin (1981), Reay et al. (1984), Gathier \& Pottasch (1988, 1989) and Jacoby \& Kaler (1989): the Zanstra discrepancy is reversed in the central star of NGC 6565, being $T_{\mathrm{Z}} \mathrm{HI}>T_{\mathrm{Z}} \mathrm{HeII}$.

The stellar luminosities are $\log L / L_{\odot}\left(T_{\mathrm{Z}} \mathrm{HI}\right)=2.3$ $( \pm 0.3)$ and $\log L / L_{\odot}\left(T_{\mathrm{Z}} \mathrm{HeII}\right)=2.0( \pm 0.3)$ (using the bolometric corrections by Schönberner 1981).

The low luminosity and high temperature of the star, combined with the short age of the nebula, suggest that the stellar mass, $M_{*}$, is larger than the average value $\left(\simeq 0.60 M_{\odot}\right)$ of the PNe nuclei (Schönberner 1979, 1981, 1983; Vassiliadis \& Wood 1993, 1994; Blöcker 1995). The quantification of $M_{*}$ through the comparison with theoretical evolutionary tracks reported in the literature gives no solutions for the He-burning post-AGB stars (these models do not predict a fast luminosity decline), whereas for the H-burning post-AGB stars different values are obtained:

$M_{*}>0.70 M_{\odot}$ (Schönberner 1981, 1983; Iben 1984),

$M_{*}>0.90 M_{\odot}($ Wood \& Faulkner 1986; Vassiliadis \& Wood 1994),

$M_{*} \simeq 0.65 M_{\odot}($ Blöcker \& Schönberner 1990; Blöcker 1995).

These discrepancies reflect the different choices by the authors of:

- the semi-empirical AGB mass-loss law, controlling both the evolution along the upper AGB and the transition into the PNe region,

- the residual, hydrogen rich envelope (and its correlation with the thermal-pulse cycle phase), and the fast
post-AGB mass-loss, determining the horizontal crossing of the HR diagram,

- the treatment of the gravo-thermal energy release and neutrino energy losses, defining the fading to the white dwarf regime.

Although a deep analysis of the central star evolution is beyond the aim of this paper, our observational data suggest the following, qualitative picture: the massive progenitor of NGC 6565 underwent a long AGB phase (favoring the degeneration of the core), a strong superwind leaving a small, hydrogen rich envelope (causing a rapid horizontal crossing), and a quite fast final fading towards the white dwarf domain (highly degenerated core and neutrino losses).

According to the critical analysis by Blöcker \& Schönberner (1990), Tylenda \& Stasinska (1994) and Blöcker $(1995)$, we assume $M_{*}($ NGC 6565$) \simeq 0.65 M_{\odot}$.

\section{The radial ionization structure}

The detailed knowledge of the plasma diagnostics in the (fully ionized + transition) zone of NGC 6565 allows us to solve the equations of statistical equilibrium, thus obtaining the point to point ionic concentrations from the line intensities (Peimbert \& Torres-Peimbert 1971; Barker 1978; Aller 1984; Osterbrock 1989).

The absolute emission profiles $\left(\mathrm{erg} \mathrm{cm}^{-3} \mathrm{~s}^{-1}\right)$ in the zvpc at $\mathrm{PA}=0^{\circ}$ and $\mathrm{PA}=90^{\circ}$ are shown in Fig. 10.

All the main ionic species are well represented in the echellograms, with the exception of $\mathrm{O}^{+}$, whose principal emissions ( $\lambda 3726 \AA$ and $\lambda 3729 \AA)$ fall outside our spectral 
range. Thus, we are forced to derive the $\mathrm{O}^{+} / \mathrm{H}^{+}$abundance from the $\lambda 7319.92 \AA / \mathrm{H} \alpha$ ratio, where $\lambda 7319.92 \AA$ is the strongest line of the red $\mathrm{O}^{+}$quartet, whose relative intensities are practically constant in the density range here considered (De Robertis et al. 1985). Since the ratio $\lambda 7319.92 \AA /(\lambda 3726 \AA+\lambda 3729 \AA)$ is in the range 0.015 to 0.075 , mainly depending on $N$ e (Keenan et al. 1999), the $\mathrm{O}^{+} / \mathrm{H}^{+}$abundance is obtained only at, or close to, the line peaks. Also, the unfavorable position of $\lambda 3967 \AA$, at the extreme blue edge of the frame, makes the quantitative $\mathrm{Ne}^{++} / \mathrm{H}^{+}$analysis quite uncertain.

At this point some caveats are in order:

1) Because of the luminosity drop of the fast evolving central star, the ionization and thermal structure of NGC 6565 are out of equilibrium: with time the $\mathrm{H} \alpha$ peak recedes (the ionized nebula zooms out) and the recombination processes prevail. They are faster in the high ionized species; this explains, at least qualitatively, the reversion of the Zanstra discrepancy reported in Sect. 7.4: $T_{\mathrm{Z}} \mathrm{HI}$ is larger than $T_{\mathrm{Z}} \mathrm{HeII}$ because $\mathrm{H} \beta$ maintains a "longer memory" than $\lambda 4686 \AA$ of the stellar luminosity.

Actually, the post-AGB evolution slows down and the luminosity gradient rapidly decreases while approaching the white dwarf domain (Schönberner 1979, 1981, 1983; Wood \& Faulkner 1986; Vassiliadis \& Wood 1993, 1994; Blöcker 1995). Because of the high gas density we can consider NGC 6565 in quasi- equilibrium: the $\mathrm{H}^{+}$recombination rate, $\mathrm{dH}^{+} / \mathrm{d} t \propto-N \mathrm{e}^{2}$, is large enough to ensure a short "relaxation" of the main nebula (the recombination time, $t_{\mathrm{rec}}(\mathrm{yr})=1 / \alpha_{\mathrm{B}} N \mathrm{e} \simeq$ $1.2 \times 10^{5} / N$ e, is a few dozen years; $\alpha_{\mathrm{B}}=$ effective recombination coefficient of hydrogen).

2) No correction for seeing is applied to the observed emission line profile; although this causes some inaccuracy for the sharpest radial distributions (like [OI], [NII] and $[\mathrm{SII}]$ ), it doesn't modify the general results here obtained.

3) No precise information is yet available for the electron temperature of the patchy, almost neutral gas in the external part of the "transition" zone, where the recombination and cooling processes dominate. This mainly concerns the $[\mathrm{OI}] \lambda 6300 \AA$ emission, which is much more sensitive to the physical conditions than are the $[\mathrm{NII}]$ and $[\mathrm{OII}]$ lines (Williams 1973). Thus, we are forced to consider different scenarios. Three possible solutions are given for $\mathrm{O}^{0} / \mathrm{H}^{+}$, corresponding to $T \mathrm{e}($ neutral $)=7500 \mathrm{~K}, T \mathrm{e}($ neutral $)=5000 \mathrm{~K}$ and $T \mathrm{e}($ neutral $) \simeq T \mathrm{e}($ ionized $)$.

Figure 11 shows the resulting radial ionization structure of NGC 6565 at $\mathrm{PA}=0^{\circ}$ (top) and $\mathrm{PA}=90^{\circ}$ (bottom). Only two elements (i.e. helium and oxygen) present an almost complete ionization structure. Note that hydrogen is well represented in the main nebula, but the lack of the neutral term appears problematic in the outermost regions, where $\mathrm{H}^{\circ}$ abounds (see Sects. 6.1 and 7.3).
This is confirmed by the $\left(\mathrm{He}^{+} / \mathrm{H}^{+}+\mathrm{He}^{++} / \mathrm{H}^{+}\right)$radial trend: it is approximately constant $(0.105 \pm 0.005)$ up to the "transition" zone and later it increases outwards. Such a behavior is typical of an optically thick PN ionized by a high temperature central star (the $\mathrm{He}^{+}$region extending further than the $\mathrm{H}^{+}$one; Hummer \& Seaton 1964; Alexander \& Balick 1997). Previous He/H abundances reported in the literature for NGC 6565 (mean values over the whole nebula) span in the range 0.095 to 0.108 (Aller et al. 1988; Köppen et al. 1991; de Freitas Pacheco et al. 1992).

\subsection{Ionization structure for $T e($ neutral $) \simeq T e$ (ionized)}

This corresponds to the common assumption of using a single temperature (Te[OIII] and/or Te[NII]) all over the nebula, even in the regions where recombination starts to be significant.

In this case the value $\left(\mathrm{O}^{0} / \mathrm{H}^{+}+\mathrm{O}^{+} / \mathrm{H}^{+}+\mathrm{O}^{++} / \mathrm{H}^{+}\right)$ is quite constant across the nebula (but in the innermost regions, where $\mathrm{O}^{+3}$ and even higher ionization stages dominate). $\mathrm{O} / \mathrm{H}$ results $6.0( \pm 1.0) \times 10^{-4}$, to be compared with the values of $5.9 \times 10^{-4}, 8.7 \times 10^{-4}$ and $10.9 \times 10^{-4}$ reported by Aller et al. (1988), Köppen et al. (1991) and de Freitas Pacheco et al. (1992), respectively.

The most striking feature of Fig. 11 is the low $\mathrm{O}^{0} / \mathrm{H}^{+}$ abundance in the outer regions of NGC 6565. This is a direct consequence of the "large" value adopted for $T$ e(neutral), favoring the emissivity of the forbidden line $\lambda 6300 \AA$ with respect to $\mathrm{H} \alpha$. The $\mathrm{O}^{0} / \mathrm{H}^{+}$underabundance indicates a scarce efficiency of the chargeexchange reaction $\mathrm{O}^{+}+\mathrm{H}^{0} \leftrightarrows \mathrm{O}^{0}+\mathrm{H}^{+}$(Williams 1973; Aller 1984; Osterbrock 1989), implying that $\mathrm{H}^{+} \gg \mathrm{H}^{0}$ (in contradiction with the result just obtained from $\mathrm{He}$ ).

All this is likely due to the unrealistic assumption $T \mathrm{e}($ neutral $) \simeq T \mathrm{e}($ ionized $)$, for the following reasons:

- the low value of $\mathrm{O}^{0} / \mathrm{H}^{+}$in the outer regions of NGC 6565 disagrees with a number of observational evidences mentioned before, indicating the presence of a large amount of neutral, dusty and patchy gas around the ionized nebula;

$-\mathrm{H}^{+} \gg \mathrm{H}^{0}$ means that the nebula is almost optically thin to the UV flux of the star, i.e. the total nebular mass is close to the ionized nebular mass $\left(M_{\text {tot }} \simeq\right.$ $\left.M_{\text {ion }} \simeq 0.03 M_{\odot}\right)$. Thus, NGC 6565 should be an undermassive nebula ejected and excited by a massive central star, contradicting the theoretical and observational evidences of a direct relation between $M_{\text {neb }}$ and $M_{*}$ (Schönberner 1981; Pottasch 1983; Vassiliadis \& Wood 1994; Boffi \& Stanghellini 1994; Blöcker 1995; Buckley \& Schneider 1995; Dopita et al. 1996).

\subsection{Ionization structure for $T e$ (neutral) $<T e$ (ionized)}

Lowering $T$ e(neutral), $\mathrm{O}^{0} / \mathrm{H}^{+}$increases in the outermost part, suggesting that charge-exchange reactions dominate in this $\mathrm{H}^{0}$-rich region (see Fig. 11). 


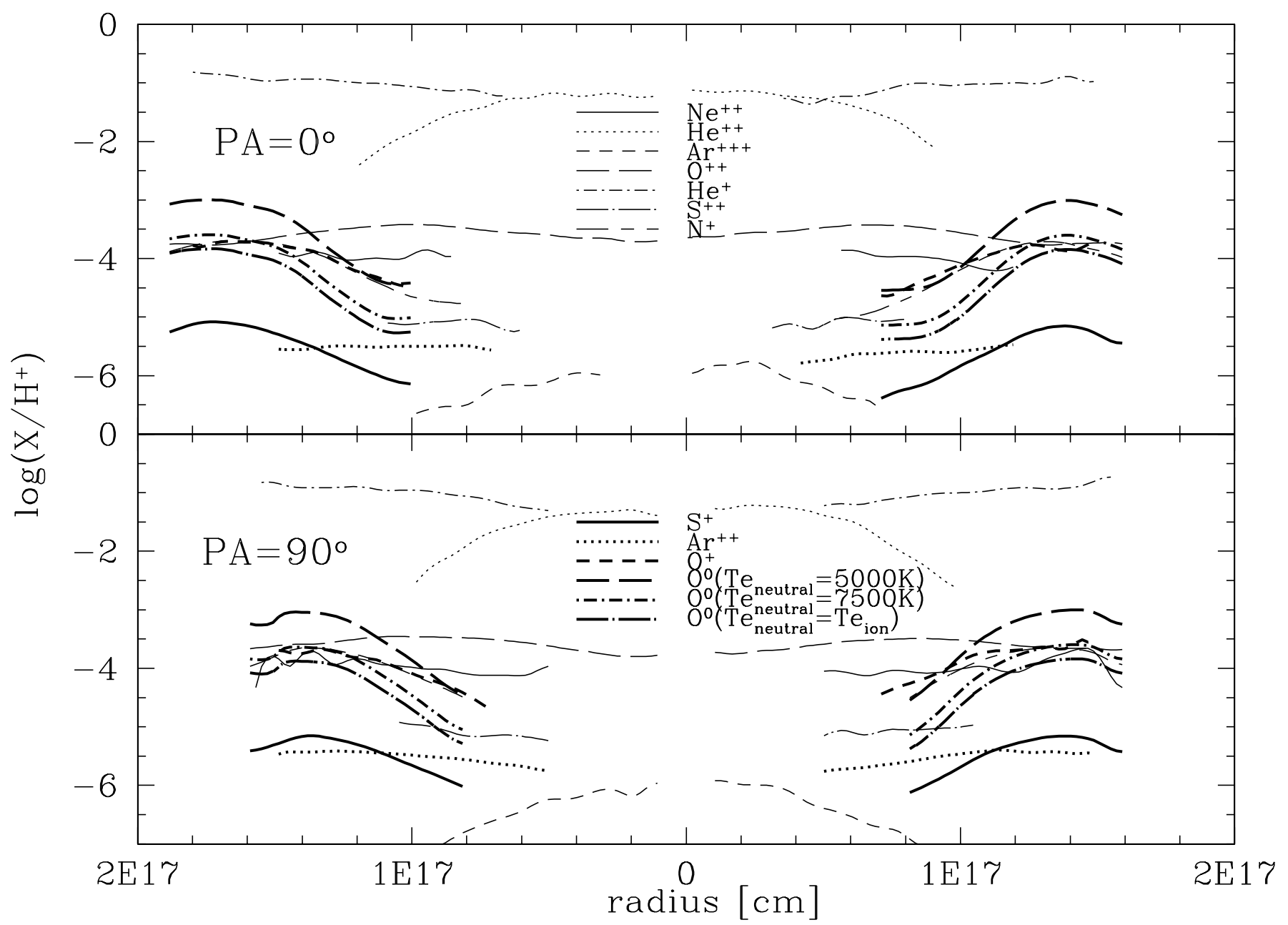

Fig. 11. The radial ionization structure of NGC 6565 at $\mathrm{PA}=0^{\circ}$ (top) and $\mathrm{PA}=90^{\circ}$ (bottom). Three solutions are shown for $\mathrm{O}^{0} / \mathrm{H}^{+}$, corresponding to $T \mathrm{e}($ neutral $)=5000 \mathrm{~K}, 7500 \mathrm{~K}$ and $\simeq T \mathrm{e}($ ionized $)$. The orientation is as in Fig. 9.

The density profile of $\mathrm{H}^{+}$has been derived all over the nebula in Sect. 6.4. The $\mathrm{H}^{0}$ concentration in the "transition" zone is difficult to estimate. An indication can be obtained as follows.

In the general form, the total abundance (relative to hydrogen) of an element $\mathrm{X}$ with atomic number $n$ is given by:

$\frac{\mathrm{X}}{\mathrm{H}}=\frac{\sum_{\mathrm{i}=0}^{n} \mathrm{X}^{i}}{\sum_{\mathrm{j}=0}^{1} \mathrm{H}^{j}}$.

For oxygen, neglecting the higher ionization terms, this can be written as:

$\frac{\mathrm{O}}{\mathrm{H}}=\frac{\mathrm{O}^{\circ}+\mathrm{O}^{+}+\mathrm{O}^{++}}{\mathrm{H}^{\circ}+\mathrm{H}^{+}}$.

Assuming $\mathrm{O} / \mathrm{H}=$ constant across the nebula, we obtain for each radial position:

$\frac{\mathrm{H}^{\circ}+\mathrm{H}^{+}}{\mathrm{H}^{+}} \simeq \frac{\frac{\mathrm{O}^{\circ}}{\mathrm{H}^{+}}+\frac{\mathrm{O}^{+}}{\mathrm{H}^{+}}+\frac{\mathrm{O}^{++}}{\mathrm{H}^{+}}}{\frac{\mathrm{O}}{\mathrm{H}}}$

which provides the $\mathrm{H}^{0}$ distribution in the "transition" zone of NGC 6565 , since O/H is derived from the "fully ionized" nebula.
The impasse caused by the uncertainties on $\mathrm{O}^{\circ} / \mathrm{H}^{+}$, due to $T$ e(neutral), can be overcame by linking the "transition" zone with the "halo", the outermost, very low electron density region which is in a recombination phase since the "recent" luminosity drop of the star.

The question is: how recent? A lower limit of about 100 years comes from the Curtis (1918) description of NGC 6565 ("No central star can be seen" with the $0.91 \mathrm{~m}$ Crossley Reflector). Moreover, the value $M_{*} \simeq 0.65 M_{\odot}$ adopted for the stellar mass (see Sect. 7.4) suggests that the luminosity drop indicatively started 1000 years ago. This must be considered as an upper limit, since the thinthick transition did not occur at the beginning of the stellar drop, but during the decline (the exact moment depending on the nebular mass; for $M_{\text {neb }} \simeq 0.15 M_{\odot}$ the thin-thick transition started at $\log L_{*} / L_{\odot} \simeq 2.8$, i.e. about 400 years ago).

More precise information comes from the observed density profile in the "transition zone" and in the "halo". The $N$ e depletion rate for recombination is given by:

$\mathrm{d} N \mathrm{e} / \mathrm{d} t=-\alpha_{\mathrm{B}} N \mathrm{e} N\left(\mathrm{H}^{+}\right)$. 


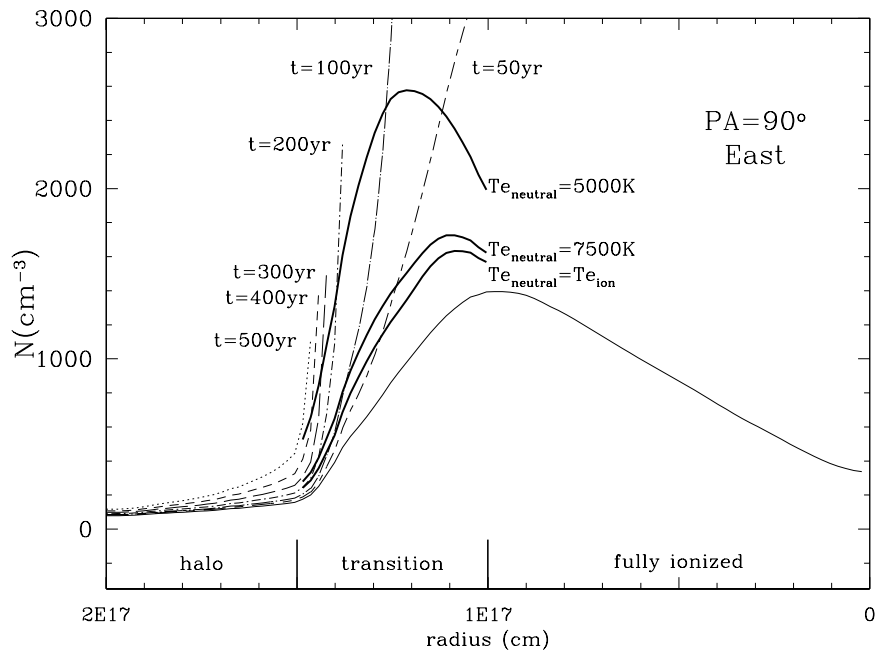

Fig. 12. Reconstruction of the (ionized +neutral) radial density profile in NGC 6565 at $\mathrm{PA}=90^{\circ}$ East sector. The orientation is as in Fig. 9. Thin continuous line: the observed $N \mathrm{e} \simeq N\left(\mathrm{H}^{+}\right)$ profile (from the $\mathrm{H} \alpha$ flux in the zvpc), for $\epsilon_{1}=1.0$. Thick continuous lines: the $N\left(\mathrm{H}_{\text {tot }}\right)$ distribution in the "transition" zone (from Eq. (8)) for three values of Te(neutral). Dashed and dotted thin lines: the $N\left(\mathrm{H}_{\text {tot }}\right)$ profile in the "transition zone" and in the "halo" for some representative values of time elapsed from the start of the recombination (from Eq. (10)).

Assuming $N \mathrm{e} \simeq N\left(\mathrm{H}^{+}\right)$and $T \mathrm{e}=10000 \mathrm{~K}$, and neglecting the recombination delay due to expansion, we obtain:

$N\left(\mathrm{H}_{\text {tot }}\right)=N \mathrm{e}(t) /\left[1-8.2 \times 10^{-6} t N \mathrm{e}(t)\right]$

which provides $N\left(\mathrm{H}_{\text {tot }}\right)$, the density in the "transition zone" and in the "halo" at the start of the recombination phase, once is known the present $N \mathrm{e}(t)$, the electron density at time $t$ (in years) elapsed from the beginning of the recombination process.

This is summarized in Fig. 12 for $\mathrm{PA}=90^{\circ}$ East sector, i.e. along the apparent minor axis of NGC 6565. It shows:

- the whole $N \mathrm{e} \simeq N\left(\mathrm{H}^{+}\right)$profile in the zvpc (from the $\mathrm{H} \alpha$ flux, for $\left.\epsilon_{\mathrm{l}}=1.0\right)$; according to the discussion in Sect. 6.5, we adopt $N e_{\text {halo }}=N e_{\text {halo }}($ Fig. 9) $/ 2$,

- the $N\left(\mathrm{H}_{\text {tot }}\right)$ distribution in the "transition" zone for $T \mathrm{e}($ neutral $) \simeq T \mathrm{e}($ ionized $), 5000 \mathrm{~K}$ and $7500 \mathrm{~K}$ (from Eq. (8)),

- the density profile, $N\left(\mathrm{H}_{\text {tot }}\right)$, in the "transition zone" and in the "halo" for some representative values of $t$ (from Eq. (10)).

In spite of the heavy assumptions (in particular: $\alpha_{\mathrm{B}}$ is a function of $T$ e and $\epsilon_{1}<1.0$ in the real nebula), Fig. 12 suggests that:

- the recombination of the main nebula indicatively started about 400 years ago;

- the nebular mass is at least $0.10 M_{\odot}$, hence most of the gas is presently neutral $\left(M_{\text {ion }}=0.03 \pm 0.01\right.$, Sect. 7.3$)$;

- the electron temperature in the external, almost neutral regions is lower than in the "ionized" nebula $(5000 \mathrm{~K}<$ Te(neutral) $<7500 \mathrm{~K})$.
Note that the chronological sequence defined by the intersection of the different curves in the "transition zone" underestimates $t$ for $t<100-200$ years, since we have neglected the contribution of the photoionization in the innermost regions of the "transition zone". This mainly concerns the $t=50$ yr curve, which considerably shifts to the right.

As already noticed in Sect. 6.1, an unrealistic situation occurs for $T \mathrm{e}($ neutral $) \simeq 5000 \mathrm{~K}$ : the complete $\mathrm{H} \alpha / \mathrm{H} \beta$ distribution observed in the spectral maps is due to $T$ e radial variations. This implies that $c(\mathrm{H} \beta)_{\text {local }} \simeq 0$ and $\mathrm{H}^{+} \gg \mathrm{H}^{0}$ (an evident nonsense stressing the urgency of a more sophisticate photo-ionization model).

The overall (fully ionized+transition+halo) structure of NGC 6565 closely recalls the recombination phase of Tylenda's $(1983,1986)$ models: only the innermost nebula is ionized by the fading UV luminosity of the star, whereas the outermost part, unattainable by the direct radiation, simply "remembers" the UV flux received just before the luminosity drop. In this scenario, the three-zones division scheme proposed in Sect. 6.5 marks two distinct evolutionary times of the star: the "fully ionized" nebula refers to the present low luminosity phase, the "halo" to its past glory.

The "transition" zone is a special case; on the whole it is recombining, but the process has been gradual (the outermost regions are the first to recombine), and decelerated (the luminosity gradient of the star decreases in time). Moreover, following Tylenda (1986) and Marten \& Szczerba (1997) the high ionization species recombine faster than the low ones, and for a given ion, the shorter the wavelength of the transition, the faster is the decline of the respective emission.

The electron temperature behavior in the "transition" zone reflects the rate of change in kinetic energy of (ions+atoms+electrons) and is the net result of two contrasting processes: the recombinations favor an increase of $T$ e since they tend to remove slow electrons, whereas the losses by forbidden lines lower Te (Aller 1984). All this, added to the inhomogeneous structure of the outermost layers, makes the situation quite complex. Following the time dependent ionization calculations by Harrington \& Marionni (1976), Tylenda (1986) and Marten \& Szczerba (1997), the electron temperature gradually decreases in time. In short, the "transition" zone constitutes a spatial and temporal link between the outer edge the "fully ionized" nebula and the recombining "halo".

The contracting phase of NGC 6565 will last only a few hundred years: with time, the dilution factor due to the expansion will overcome the slower and slower stellar decline, thus leading to a gradual re-growing of the ionization front.

Actually, we cannot completely rule out the possibility that the re-growing already takes place in NGC 6565. According to the classical Strömgren model of nebular ionization, the contraction-expansion equilibrium occurs for $-1 / 3 \mathrm{~d}\left(\ln L_{*}\right) / \mathrm{d} t=2 / t_{\mathrm{dyn}}$, i.e. it depends on the present rate of luminosity decline of the star. 
Table 2. Total chemical abundances in NGC 6565 .

\begin{tabular}{l}
\hline $\mathrm{He} / \mathrm{H}=0.105( \pm 0.003)$ \\
$\mathrm{O} / \mathrm{H}=6.0( \pm 1.0) \times 10^{-4}$ \\
$\mathrm{~N} / \mathrm{H}=1.5( \pm 0.3) \times 10^{-4}$ \\
$\mathrm{Ne} / \mathrm{H}=1.6( \pm 0.4) \times 10^{-4}$ \\
$\mathrm{~S} / \mathrm{H}=6.3( \pm 1.0) \times 10^{-6}$ \\
$\mathrm{Ar} / \mathrm{H}=1.8( \pm 0.4) \times 10^{-6}$ \\
\hline
\end{tabular}

The evolutionary tracks for hydrogen burning post-AGB stars by Schönberner (1981), Wood \& Faulkner (1986), Vassiliadis \& Wood (1994) and Blöcker (1995) give contrasting results.

Although a clear answer needs first and second epoch HST imagery in the main nebular emissions, our feeling is that NGC 6565 is still contracting, because of the observed reversion of the Zanstra discrepancy (or, at least, it seems to be still contracting, due to the time delay in the nebular adjustement to the changing UV luminosity of the star).

We conclude this Section with the total chemical abundances (Table 2). They are derived in the usual way, i.e. multiplying the observed ionic abundances for the corresponding ICFs, the correcting factors for the unobserved ionic stages (obtained both empirically, Barker 1983, 1986; and from interpolation of theoretical nebular models, Shields et al. 1981; Aller \& Czyzak 1983; Aller 1984; Osterbrock 1989). Following the critical analysis by Alexander \& Balick (1997), we have considered the total line intensities (i.e. integrated over the whole spatial profile and the expansion velocity field).

In general, the chemical abundances of Table 2 are in satisfactory agreement with the values reported in the literature (Aller et al. 1988; Köppen et al. 1991; de Freitas Pacheco et al. 1992), but for the $N$ overabundance suggested by Aller et al. (1988) which is not confirmed here.

\section{The photo-ionization model}

In order to test the results obtained for the physical conditions and the ionization structure, and to disentangle the controversial points of the foregoing Sections, we have applied the photo-ionization code CLOUDY (Ferland et al. 1998) to a mass of gas having the same density distribution and chemical composition of our $\mathrm{PN}$, and a powering source similar to the central star of NGC 6565.

The usual caveat: CLOUDY is a "steady-state" model. It works in the "fully ionized" part of NGC 6565, which is in quasi-equilibrium (see Sect. 8), but it cannot depict the external, recombining regions originated by the luminosity drop of the central star.

We consider only a single sector of a single PA (East sector of $\mathrm{PA}=90^{\circ}$, i.e. along the apparent minor axis) as representative of the whole equatorial regions of NGC 6565 identified by the zvpc.

The input parameters of the model nebula are given in Table 3 . The adopted $N\left(\mathrm{H}_{\text {tot }}\right)$ profile corresponds to the "fully ionized" part of $N\left(\mathrm{H}^{+}\right)$at $\mathrm{PA}=90^{\circ}$ East sector, for
Table 3. Input parameters of the model nebula.

\begin{tabular}{ll}
\hline Radial density profile & $\begin{array}{l}N\left(\mathrm{H}_{\text {tot }}\right) \text { in top panel of Fig. 13 } \\
\text { (cf. Sect. 8.2) }\end{array}$ \\
Filling factor & 1 \\
Chemical abundances: & \\
C, Si, Cl & Aller et al. (1988) \\
He, N, O, Ne, S, Ar & this paper \\
other elements & PN (CLOUDY default) \\
Dust & PN (CLOUDY default) \\
Star & blackbody with $T_{*}=120000 \mathrm{~K}$ and \\
& $\log L_{*} / L_{\odot}=2.0$ \\
\hline
\end{tabular}

$\epsilon_{1}=1$ (see Fig. 9). It is arbitrarily extended to the "transition" and the "halo" in order to obtain a nebular mass of about $0.30 M_{\odot}$. The choice of different $N(\mathrm{H})$ profiles in the outermost regions does not modify the resulting ionization structure, since these layers cannot be reached by the UV stellar flux. Concerning the ionizing source, we use a blackbody distribution having the Zanstra HeII temperature and luminosity of the central star of NGC 6565 (see Sect. 7.4). Once it is convolved for a seeing of 0.65 arcsec, the line emissivity (per unit filling factor) of the model nebula at $2.0 \mathrm{kpc}$ can be compared with the emission per unit volume presented in Fig. 10. The results are shown in Fig. 13.

The steady-state model nebula convolved for seeing (third panel in Fig. 13) closely reproduces the "fully ionized" part of NGC 6565 (bottom panel) up to $R \simeq 1.0 \times$ $10^{17} \mathrm{~cm}$. An even better fit might be obtained through a modest adjustment of the chemical abundances. The discrepancies begin in the "transition" zone and increase outward ("halo").

The comparison of the model vs. observed nebula offers an encouraging support to the results obtained in the previous sections. In particular, Fig. 13 confirms the peculiar properties of $[\mathrm{ArIV}]$ at $\lambda 4740 \AA$, as pointed out in Sect. 4: the structure of $\mathrm{Ar}^{+3}$ (I.P. $=40.7 \mathrm{eV}$ ) mimics that of higher ionization species since it is present in the interval (40.7-59.8) eV, the upper limit being the ionization potential of $\mathrm{Ar}^{+4}$, i.e. it overlaps the $\mathrm{He}^{++}$zone.

Neon shows the opposite behaviour of Argon: $\mathrm{Ne}^{++}$ is expected to within the $(40.9-63.4) \mathrm{eV}$ range, whereas in Fig. 13 the [NeIII] emission at $\lambda 3967 \AA$ extends to the medium-low ionization region (see also Sect. 4 and Figs. 5 and 10). We have verified on a number of model nebulae: the abnormal extension of $\mathrm{Ne}^{++}$only occurs in the presence of a hot central star. According to Williams (1973), Butler et al. (1980), Shields et al. (1983) and Clegg et al. (1986), a large number of stellar photons with energies well above the HI ionization threshold maintains a high degree of ionization in the nebula right up to the point where $\mathrm{H}^{\circ}$ prevails, and the charge-exchange reaction $\mathrm{Ne}^{+3}+\mathrm{H}^{\circ} \leftrightarrows \mathrm{Ne}^{++}+\mathrm{H}^{+}$populates the ${ }^{5} \mathrm{P},{ }^{3} \mathrm{D}^{\circ}$ and ${ }^{3} \mathrm{P}$ levels of $\mathrm{Ne}^{++}$. The ground state is then reached via radiative decay, and the collisionally excited lines at $\lambda 3868 \AA$ and $\lambda 3967 \AA$ are emitted. 


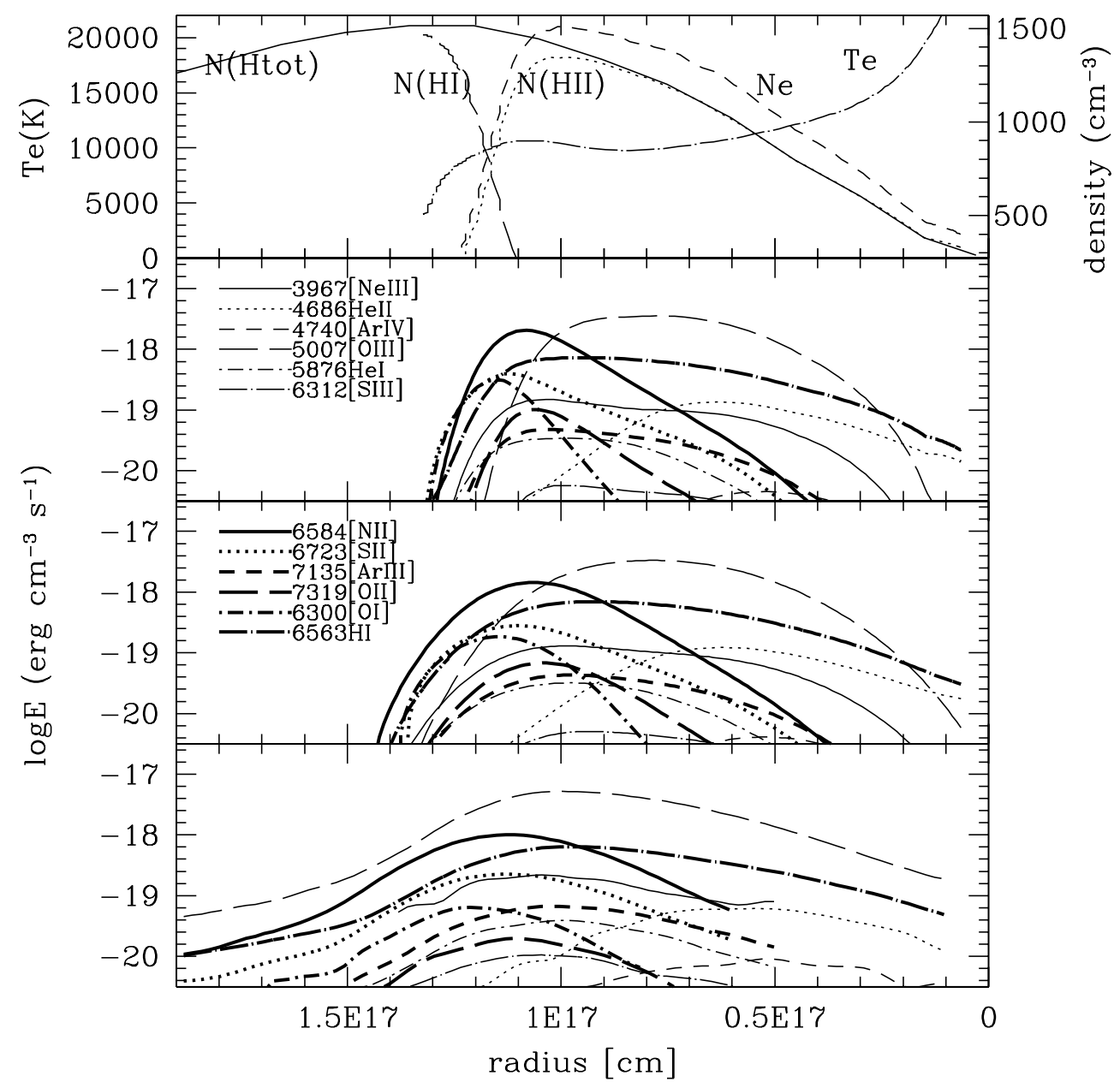

Fig. 13. Model nebula vs. NGC 6565 at $\mathrm{PA}=90^{\circ}$, East sector. Top panel: radial profiles of $T$ e (left ordinate scale) and $N$ e, $N\left(\mathrm{H}^{+}\right), N\left(\mathrm{H}^{\circ}\right)$ and of the input density $N\left(\mathrm{H}_{\text {tot }}\right)$ (right ordinate scale) for the model nebula in the case $\epsilon_{1}=1.0$. Second panel: absolute radial flux distribution $\left(\mathrm{erg} \mathrm{s}^{-1} \mathrm{~cm}^{-3}\right.$ ) of the model nebula in the main emissions. Third panel: same as in the second panel, after convolution for a seeing of 0.65 arcsec, reproducing the observational conditions of the true nebula. Bottom panel: absolute flux distribution in the main emissions of NGC 6565 at PA $=90^{\circ}$, East sector (cf. Fig. 10).

Concerning the electron temperature, the radial behavior of the model nebula (Fig. 13, top panel) closely reproduces the observed one (Fig. 9, middle panel) up to the "transition" zone. The external, fast Te drop of the model nebula clearly refers to a steady-state situation, which does not take into account the presence of the broad recombining region in NGC 6565.

Notice the quantitative agreement between $E(\mathrm{H} \alpha)$ of the model nebula, convolved for seeing, and the observed one. This is a consequence of the clumpiness found in Sect. 7.2 (i.e. $\epsilon_{1} \simeq 0.25$ ). In fact, the former is obtained from the $N\left(\mathrm{H}_{\text {tot }}\right)$ profile under the assumption $\epsilon_{1}=1$, whereas in the real nebula $N\left(\mathrm{H}^{+}\right)=\epsilon_{1}^{1 / 2} \times N \mathrm{e}[\mathrm{SII}]=$ $0.5 \times N \mathrm{Ne}[\mathrm{SII}]$.

In Fig. 13 the most striking discrepancy concerns the radial $\mathrm{O}^{++}$profile: in NGC 6565 (bottom panel) it closely reproduces the $\mathrm{H}^{+}$one, as expected of an optically thick nebula powered by a high temperature central star (Flower 1969; Williams 1973), while in both the model nebula and the convolved model nebula (second and third panel, respectively) $\mathrm{H}^{+}$extends further than $\mathrm{O}^{++}$and the outermost ionized regions are $\mathrm{O}^{++}$-depleted.

We stress that the application of the photo-ionization code CLOUDY to the other PA of NGC 6565 gives results coinciding with the ones just presented for $\mathrm{PA}=90^{\circ}$, East sector.

We conclude that the nebular modeling is a powerful instrument for interpreting the observed properties of each PN. In the specific case of NGC 6565, the nebula is so peculiar that it cannot be completely reproduced even by a detailed and sophisticate code like CLOUDY. An evolving model is required, which takes into account the nebular reaction to the rapidly changing UV luminosity of the star.

NGC 6565 is not a unique case. The recombination phase must be quite common in $\mathrm{PNe}$, almost ineluctable in the presence of a massive, i.e. fast evolving, central star. Tylenda (1986) lists a dozen well-studied objects, including the famous NGC 7027, NGC 7293, NGC 6853 and NGC 6720, and Corradi et al. (2000) suggest that a recombining halo is present in NGC 2438, but many other 
candidates are contained in the Acker et al. (1992) and Kohoutek (2000) catalogues on the basis of their optical appearance, spectroscopic characteristics and star faintness. Notice that the combination of large optical extension and high surface brightness makes the Ring nebula the ideal laboratory for analyzing in detail the recombination process in $\mathrm{PNe}$.

\section{The spatial structure}

\subsection{Tomography}

The reconstruction of the emission line structure in the nebular slices covered by the slit was introduced by Sabbadin et al. (2000a,b) and Ragazzoni et al. (2001). In the case of NGC 6565 we have selected $\lambda 4686 \AA$ of HeII, $\lambda 5007 \AA$ of $[\mathrm{OIII}]$ and $\lambda 6584 \AA$ of $[\mathrm{NII}]$ as representative of the high, mean and low ionization regions, respectively, and $\mathrm{H} \alpha$ as a marker of the whole ionized gas distribution.

The spectral images of the forbidden lines were deconvolved for seeing, spectral resolution and thermal motions, while also fine structure was considered for the recombination lines. They were de-projected using the expansion law derived in Sect. 7.2, i.e. adopting $V \exp / r=$ $5.6 \mathrm{~km} \mathrm{~s}^{-1} \operatorname{arcsec}^{-1}$. The resulting tomographic maps (quite similar to the spectral images shown in Figs. 4 and 7 ) were assembled with the procedure described by Ragazzoni et al. (2001), thus obtaining the spatial structure presented in the next section.

\subsection{3-D morpho-kinematical structure}

In order to render the 3-D structure, we adopt the method introduced by Ragazzoni et al. (2001): a series of images showing the nebula from different directions, separated by $15^{\circ}$. Each couple of images constitutes a stereoscopic pair, illuding the reader to see NGC 6565 in 3-D.

The opaque reconstruction in $\mathrm{H} \alpha$ is shown in Fig. 14 for two absolute flux cuts: $\log E(\mathrm{H} \alpha)=$ $-19.55 \mathrm{erg} \mathrm{s}^{-1} \mathrm{~cm}^{-3}$ (upper part of each panel) and $-18.50 \mathrm{erg} \mathrm{s}^{-1} \mathrm{~cm}^{-3}$ (lower part). Since $E(\mathrm{H} \alpha)=h \nu$ $\alpha_{\mathrm{H} \alpha}^{\mathrm{eff}} N$ e $N\left(\mathrm{H}^{+}\right) \epsilon_{\mathrm{l}}$, the adopted cuts correspond to $N \mathrm{e} \simeq$ $300 \mathrm{~cm}^{-3}$ (upper) and $N \mathrm{e} \simeq 1000 \mathrm{~cm}^{-3}$ (lower) (for $T \mathrm{e}=$ $10^{4} \mathrm{~K}$ and $\left.\epsilon_{\mathrm{l}}=1\right)$.

The complexity of the nebular structure in the faint, low ionization polar cups is more evident in Fig. 15, referring to $\lambda 6584 \AA$ of $[\mathrm{NII}]$ at the two cuts: log $E(\lambda 6584 \AA)=-19.40 \mathrm{erg} \mathrm{s}^{-1} \mathrm{~cm}^{-3}$ (upper part of each panel) and $-18.40 \mathrm{erg} \mathrm{s}^{-1} \mathrm{~cm}^{-3}$ (lower part). In this case $E(\lambda 6584 \AA) \propto N$ e $N\left(\mathrm{~N}^{+}\right)$, thus a precise $N$ e cut cannot be assigned: Fig. 15 simply represents the "faintest" and the "brightest" low ionization regions, respectively.

NGC 6565 in the high ionization HeII emission at $\lambda 4686 \AA$ (not shown here for reasons of space) consists of a compact, almost tubular structure recalling the $\mathrm{H} \alpha$ one (high cut) presented in Fig. 14. Moreover, the spatial form in [OIII] at $\lambda 5007 \AA$ closely reproduces the $\mathrm{H} \alpha$ one (Fig. 14), as expected of the similarity of the spectral images and the radial profiles (see Sects. 2, 4 and 8).
Finally, the distribution of the neutral gas in the transition zone of NGC 6565 (obtained from Eq. (8) using the approximation $\mathrm{H}_{\text {tot }} / \mathrm{H}^{+} \simeq \mathrm{O}^{\circ} / \mathrm{O}^{++}$and assuming $T \mathrm{e}($ neutral gas $)=6000 \mathrm{~K}$ and $T \mathrm{e}([\mathrm{OIII}])=11000 \mathrm{~K})$ mimics the $\mathrm{N}^{+}$structure shown in Fig. 15, although peaked a bit further away.

In short, NGC 6565 is an inhomogeneous triaxial ellipsoid seen almost pole-on (major axis $=10.1$ arcsec projected in $(5,7)$, intermediate axis $=7.1 \operatorname{arcsec}$ in $(-85,7)$, minor axis $=6.0$ arcsec in $(5,-83))$. The matter along and close to the major axis was swept-up by some accelerating agent (high velocity wind? ionization? magnetic fields?), forming two fast, asymmetric and faint polar cups projected in $(18,7$; i.e. they are lightly misaligned with respect to the major axis) and extending up to about 10.0 arcsec from the star. The optical nebula is embedded in a large cocoon of almost neutral gas, which is the result of the recombining processes occurred during the luminosity decline of the powering star.

The [OIII] and [NII] appearance of NGC 6565 seen from different directions is illustrated in Fig. 16. It gives a representative sample of the possible morphologies assumed by our nebula when changing the line of view, and confirms its structural similarity with NGC 6886 (suggested in Sect. 3), as well with K 3-92, M 1-7, M 1-59, M 1-66, M 2-40 and M 2-53 (see the imagery catalogues by Schwarz et al. 1992; Manchado et al. 1996; Gorny et al. 1999).

Also the observed kinematics depends on the line of view, as shown in Table 4 (upper panel). NGC 6565 can be either a compact, roundish, fast expanding object or a bipolar, elongated, slow one with all the intermediate cases. The morpho-kinematical range further spreads out in the case of a "butterfly" $\mathrm{PN}$, like $\mathrm{Hb} 5, \mathrm{Mz} 3$ and NGC 6537.

Decreasing of the spatial resolution exacerbates the problem. For example, by putting NGC 6565 at the distance of the Large Magellanic Cloud (i.e. $50 \mathrm{kpc}$; Kovacs 2000 and references therein) we obtain the results of Table 4, bottom panel. In this case the echellograms completely lose the spatial information due to the nebula compactness. $2 V \exp [\mathrm{OIII}]$ (peak separation) cannot be measured any longer (in Table 4 it has been replaced by $2 V \exp [\mathrm{OIII}](F W H M))$ and the morphological classification is quite uncertain.

All this evidenziates the difficulties implied in the analysis of the high dispersion spectra and questions the reliability of the interpretations, evolutionary correlations and spatio-kinematical models based on echellograms at inadequate spatial and spectral resolutions.

\section{Discussion and conclusions}

The long travel dedicated to the study of NGC 6565 is over. We started with the kinematical properties, passed through the physical conditions and arrived to the distance and radius of the nebula and to the temperature and luminosity of the star. The radial ionization structure was 


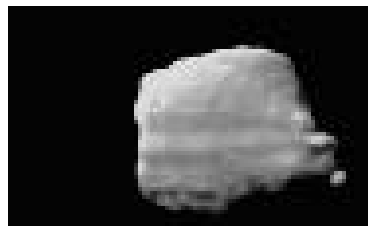

$(0,45)$
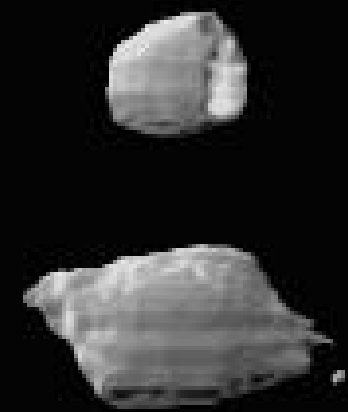

$(0,90)$
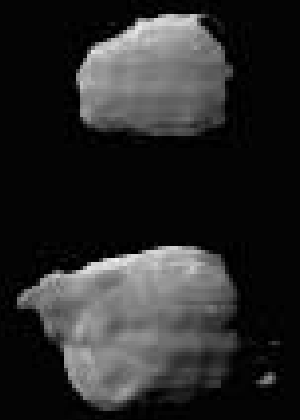

$(0,135)$
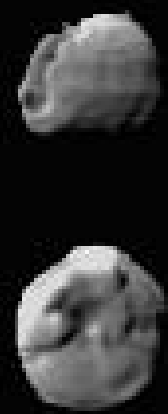

$(0,180)$

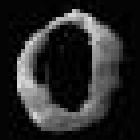

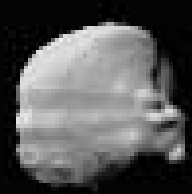

$(0,30)$
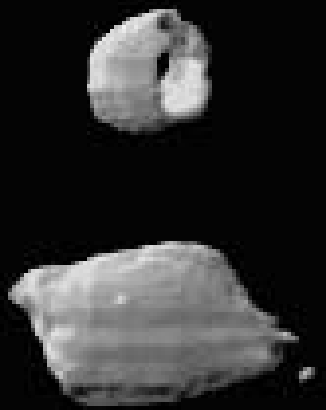

$(0,75)$
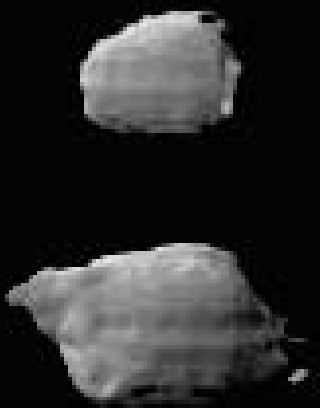

$(0,120)$
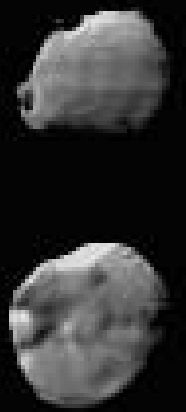

$(0,165)$

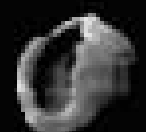

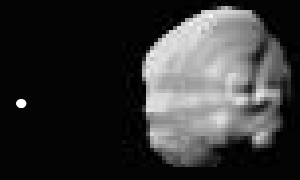

$(0,15)$
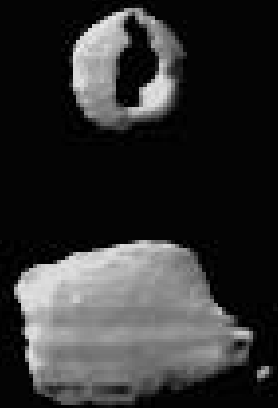

$(0,60)$
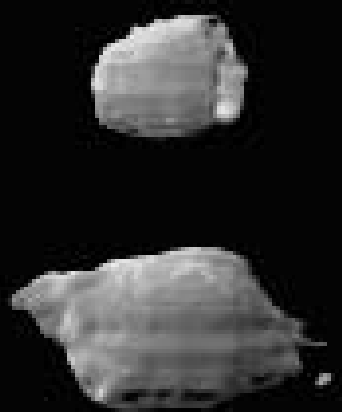

$(0,105)$
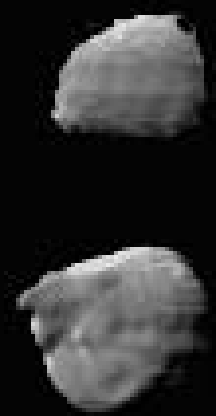

$(0,150)$

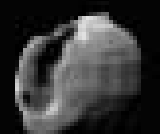

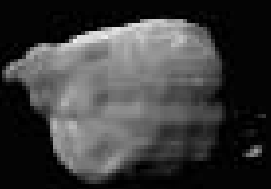

$(0,135)$

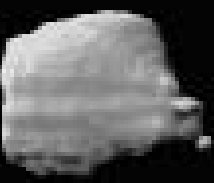

$(0,45)$
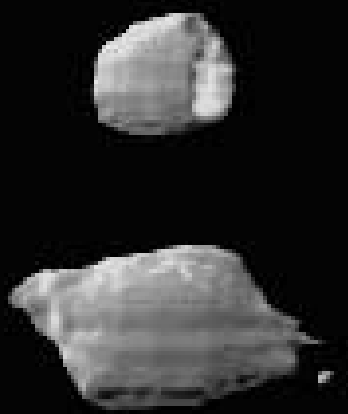

$(0,90)$
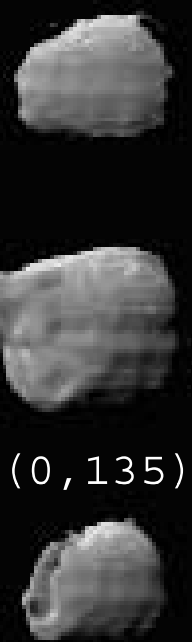

Fig. 14. Opaque reconstruction of NGC 6565 in $\mathrm{H} \alpha$ at $N \mathrm{e} \simeq 300 \mathrm{~cm}^{-3}$ (for $\epsilon_{1}=1$, upper part of each panel) and $N$ e $\simeq$ $1000 \mathrm{~cm}^{-3}$ (bottom part), as seen from 13 directions separated by $15^{\circ}$. The line of view is given by $(\theta, \psi)$, where $\theta$ is the zenith angle and $\psi$ the azimuthal angle; the upper-right image is the rebuilt-nebula seen from the Earth, i.e. from $(0,0)$. Each horizontal couple represents a "direct" stereoscopic pair, allowing the reader to have $123-\mathrm{D}$ views of the nebula in as many directions, all together covering a straight angle. We repeat here the instructions given by Ragazzoni et al. (2001): to obtain the three-dimensional vision, look at a distant object and slowly insert the figure in the field of view, always maintaining your eyes parallel. Alternatively, you can use the two small dots in the upper part of the figure as follows: approach the page till the two dots merge (they appear out of focus); then recede very slowly, always maintaining the two dots superimposed, till the image appears in focus. 


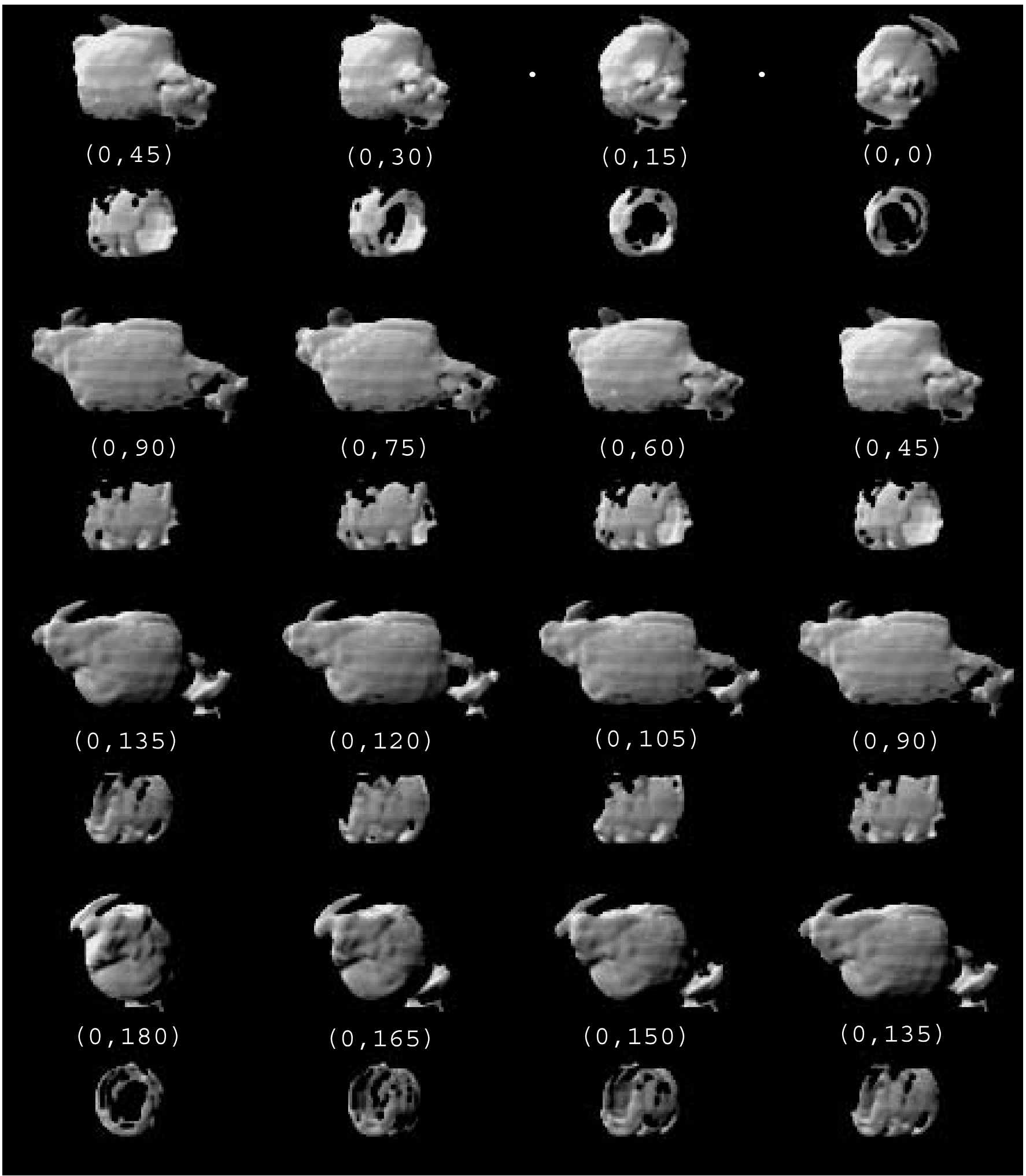

Fig. 15. Same as Fig. 14, but for [NII], showing the faintest and the brightest (upper and lower part of each panel, respectively) low ionization regions of NGC 6565 .

later explored, using both the "classical" method and the photo-ionization code CLOUDY, and the spatial structure investigated by assembling the tomographic maps.

In nuce: NGC 6565 is a young (2000-2500 yr), patchy, optically thick ellipsoid with extended polar cups, seen almost pole-on. It is in a recombination phase, because of the luminosity drop of the massive powering star, which is reaching the white dwarf domain. The stellar decline started about 1000 years ago, but the nebula remained optically thin for other 600 years before the recombination 


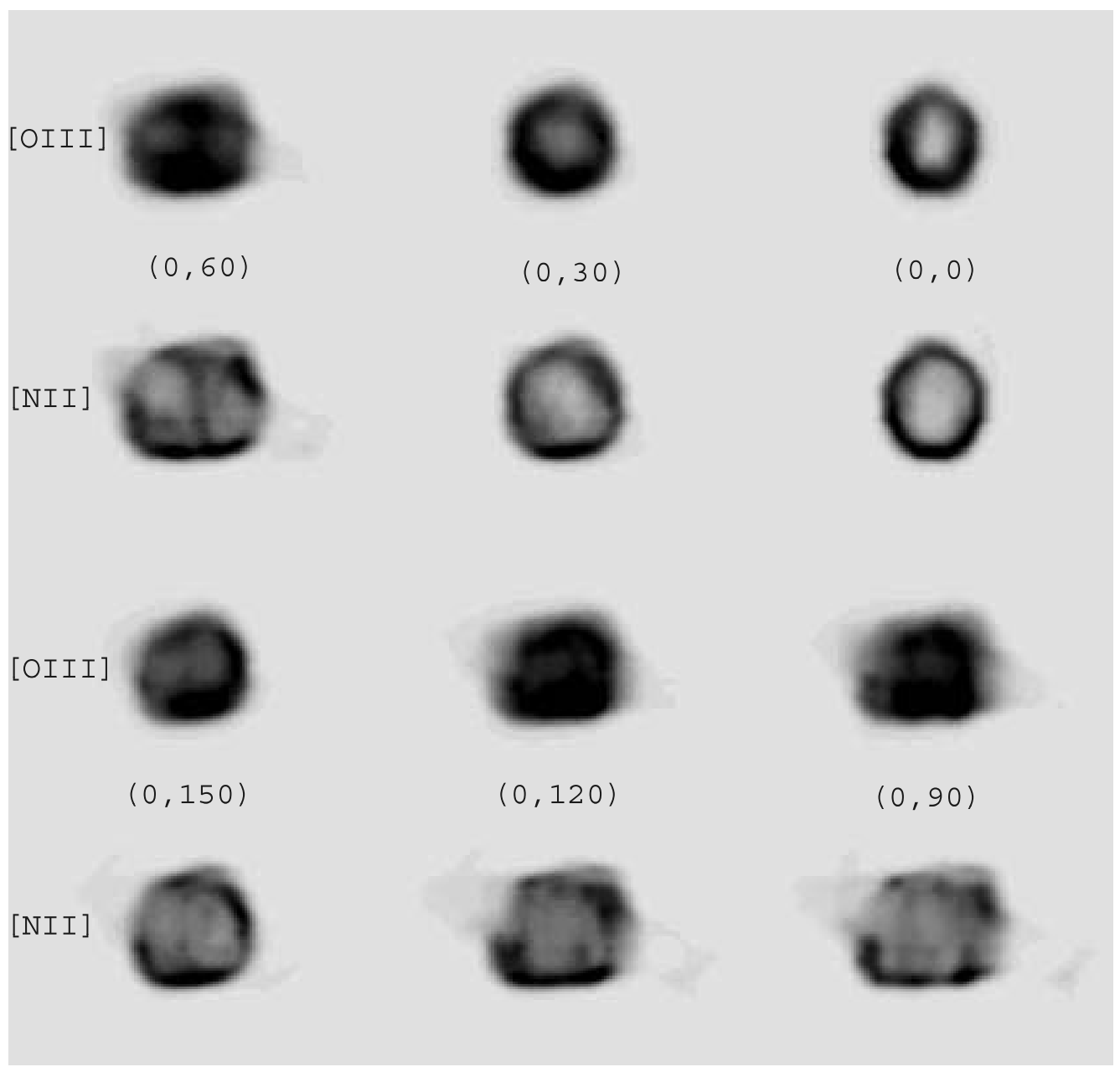

Fig. 16. [OIII] and [NII] appearance of NGC 6565 from 6 directions separated by $30^{\circ}$, showing the different morphologies when changing the line of view. The upper-right panel $(\theta, \psi=0,0)$ corresponds to the rebuilt-nebula seen from the Earth (to be compared with Fig. 1). The scale is the same of Figs. 14 and 15 . Remember that projection $(\theta, \psi)=\operatorname{projection}\left(\theta \pm 180^{\circ}\right.$, $\left.\psi \pm 180^{\circ}\right)$.

Table 4. Morpho-kinematical characteristics.

NGC 6565 at $2.0 \mathrm{kpc}$

\begin{tabular}{|c|c|c|c|c|c|c|}
\hline Projection & $\begin{array}{l}2 V \exp [\mathrm{OIII}] \\
(\text { peak } \\
{\left[\mathrm{km} \mathrm{s}^{-1}\right]}\end{array}$ & $\begin{array}{l}2 V \exp [\mathrm{NII}] \\
\text { separation }) \\
{\left[\mathrm{km} \mathrm{s}^{-1}\right]}\end{array}$ & $\begin{array}{l}2 V \exp [\mathrm{OIII}] \\
(10 \% \text { maximu } \\
{\left[\mathrm{km} \mathrm{s}^{-1}\right]}\end{array}$ & $\begin{array}{l}2 V \exp [\mathrm{NII}] \\
\mathrm{m} \text { intensity) } \\
{\left[\mathrm{km} \mathrm{s}^{-1}\right]}\end{array}$ & morphology & $\begin{array}{l}\text { apparent size } \\
{[\operatorname{arcsec}]}\end{array}$ \\
\hline $\begin{array}{l}\text { along the major axis } \\
\text { along the intermediate axis } \\
\text { along the minor axis }\end{array}$ & $\begin{array}{l}57( \pm 2) \\
42( \pm 2) \\
34( \pm 2)\end{array}$ & $\begin{array}{l}61( \pm 2) \\
46( \pm 2) \\
39( \pm 2)\end{array}$ & $\begin{array}{l}90( \pm 4) \\
50( \pm 4) \\
40( \pm 4)\end{array}$ & $\begin{array}{l}105( \pm 4) \\
77( \pm 4) \\
62( \pm 4)\end{array}$ & $\begin{array}{l}\text { elliptical } \\
\text { bipolar } \\
\text { bipolar }\end{array}$ & $\begin{array}{l}10.0 \times 8.5 \\
20.0 \times 8.5 \\
20.0 \times 10.0\end{array}$ \\
\hline & NGC 6565 & at the distance of tl & e Large Magell & nic Cloud & & \\
\hline Projection & $\begin{array}{l}2 V \exp [\mathrm{OIII}] \\
(F W H M) \\
{\left[\mathrm{km} \mathrm{s}^{-1}\right]}\end{array}$ & $\begin{array}{l}2 V \exp [\mathrm{NII}] \\
\text { (peak separation) } \\
{\left[\mathrm{km} \mathrm{s}^{-1}\right]}\end{array}$ & $\begin{array}{l}2 V \exp [\mathrm{OIII}] \\
(10 \% \text { maxim } \\
{\left[\mathrm{km} \mathrm{s}^{-1}\right]}\end{array}$ & $\begin{array}{l}2 V \exp [\mathrm{NII}] \\
\mathrm{m} \text { intensity) } \\
{\left[\mathrm{km} \mathrm{s}^{-1}\right]}\end{array}$ & morphology & $\begin{array}{l}\text { apparent size } \\
{[\operatorname{arcsec}]}\end{array}$ \\
\hline $\begin{array}{l}\text { along the major axis } \\
\text { along the intermediate axis } \\
\text { along the minor axis }\end{array}$ & $\begin{array}{l}57( \pm 4) \\
44( \pm 4) \\
36( \pm 4)\end{array}$ & $\begin{array}{l}47( \pm 4) \\
36( \pm 4) \\
31( \pm 4)\end{array}$ & $\begin{array}{l}80( \pm 4) \\
60( \pm 4) \\
48( \pm 4)\end{array}$ & $\begin{array}{l}86( \pm 4) \\
66( \pm 4) \\
55( \pm 4)\end{array}$ & $\begin{array}{l}- \\
-\end{array}$ & $\begin{array}{l}0.40 \times 0.34 \\
0.80 \times 0.34 \\
0.80 \times 0.40\end{array}$ \\
\hline
\end{tabular}


phase occurred (thus, at Galileo Galilei times NGC 6565 was at high excitation, a bit larger and much brighter than at present). In the near future the ionization front will regrow, since the dilution factor due to the expansion will prevail on the slower and slower luminosity decline.

The nebula, at a distance of $2.0 \mathrm{kpc}$, can be divided into three radial zones:

- the "fully ionized", extending up to $0.029-0.035 \mathrm{pc}$ at the equator $(\simeq 0.050 \mathrm{pc}$ at the poles $)$,

- the "transition", up to $0.048-0.054 \mathrm{pc}(\simeq 0.080 \mathrm{pc}$ at the poles),

- the recombining "halo", detectable up to $0.110 \mathrm{pc}$. The ionized mass $\left(\simeq 0.03 M_{\odot}\right)$ is only a fraction of the total mass $\left(M_{\text {tot }} \geq 0.15 M_{\odot}\right)$, which has been ejected by an equatorial enhanced superwind of $4( \pm 2) \times 10^{-5} M_{\odot} \mathrm{yr}^{-1}$ lasted for $4( \pm 2) \times 10^{3}$ years.

From all the points of view NGC 6565 results to be an exciting laboratory which deserves further attention. Amongst the many and important aspects left unresolved by this paper we mention:

- the neutral gas distribution and kinematics. Detailed radio and infrared observations are highly advisable; for instance, the spectroscopically resolved structure in the $\mathrm{H}_{2}$ emission at $2.122 \mu \mathrm{m}$, which is a signpost of the bipolar structure (Kastner et al. 1996);

- the filling factor. We obtain $\epsilon_{1}=0.25$ in the external, low ionization regions by comparing $N \mathrm{e}(\mathrm{H} \alpha)$ and $N \mathrm{e}[\mathrm{SII}]$. The same analysis should be performed in the internal, high ionization parts using [ArIV] $(\lambda 4711 \AA / \lambda 4740 \AA)$ and [ClIII] $(\lambda 5517 \AA / \lambda 5537 \AA)$. Deeper echellograms are required;

- the faint and fast (shocked?) polar cups, overlooked in our study mainly focused on the equatorial regions;

- the mechanisms and physical processes forming and shaping a PN like NGC 6565. This point appears problematic, mainly because of the huge number of models proposed for bipolar PNe.

They include: single AGB progenitors (+ fast wind + magnetic fields + photoionization), a planetary system, a binary system undergoing common envelope evolution, a close companion presenting a dense accretion disk (for details, see Frank 1999 and Soker \& Rappaport 2001).

In all these models the nebular shaping is a slow and gradual process occurring in a time scale comparable to the PN life, whereas the superior spatial resolution of the HST imagery has recently revealed that bipolarity is a common feature already in proto-PNe (see Sahai \& Trauger 1998, and references therein). This, on the one hand means that some imprint agent acts in the late AGB and/or early post-AGB phase, driving most of the subsequent nebular evolution, on the other hand stresses the inadequacy of the current models.

In addition to the specific analysis of our nebula, the aim of the present work was the search for a satisfying reduction procedure allowing us to exploit the huge amount of information contained in the high resolution spectra. They essentially cover two wide fields: kinematics (including expansion velocity, tomography and spatial structure), and physical conditions (radial profile of $\mathrm{Ne}$, $T \mathrm{e}$, ionization, chemical abundances etc.). The difficulties connected to the determination of the different parameters are perfectly synthesized in the Aller's (1994) sentence: "A nebula is a three-dimensional structure for which we obtain a two-dimensional projection". With the introduction of the 3-D methodology, the second half of the sentence becomes: "... for which we obtain the three-dimensional structure".

The accuracy of the 3-D reconstruction is defined by the "relative" spectral and spatial resolutions (Ragazzoni et al. 2001). The first is given by $R R=\Delta V / V \exp , \Delta V$ being the spectral resolution. $R R$ mainly depends on the intrumentation, since the expansion velocity range of the PNe is quite sharp (indicatively: $20 \mathrm{~km} \mathrm{~s}^{-1}<V \exp <$ $\left.40 \mathrm{~km} \mathrm{~s}^{-1}\right)$. The relative spatial resolution, $S S=d / \Delta d$ ( $d=$ angular extent, $\Delta d=$ seeing + guiding $)$, essentially depends on the target, due to the large spread of apparent sizes exhibited by the PNe. In the case of a compact object like NGC 6565, the ESO NTT+EMMI echellograms give $R R \simeq 5$ and $S S \simeq 10$; a significant increase of the spatial resolution is expected for the largest nebulae of the sample (like NGC 7009 and NGC 6818).

In conclusion, we believe that the crucial point for the physical interpretation of the PNe is the detailed knowledge of the radial density profile, now obtainable from the $\mathrm{H} \alpha$ flux distribution in the zero-velocity pixel column. It opens the possibility of creating a realistic model for each expanding nebula (this includes: PNe, nova and supernova remnants, shells around Population I Wolf-Rayet stars, nebulae ejected by symbiotic stars, bubbles surrounding early spectral type main sequence stars etc.).

Acknowledgements. We greatly appreciated the suggestions, criticisms and encouragements by Romuald Tylenda, Arsen Hajian, Gary Ferland, Detlef Schönberner and Vincent Icke.

\section{References}

Acker, A. 1978, A\&AS, 33, 367

Acker, A., Raytchev, B., Stenholm, B., \& Tylenda, R. 1991, A\&AS, 90, 89

Acker, A., Ochsenbein, F., Stenholm, B., et al. 1992, Strasbourg-ESO Catalogue of Galactic Planetary Nebulae, ESO, Garching

Alexander, J., \& Balick, B. 1997, AJ, 114, 713

Aller, L. H. 1984, Physics of Gaseous Nebulae (Reidel, Dordrecht)

Aller, L. H. 1990, PASP, 102, 1097

Aller, L. H. 1994, ApJ, 432, 427

Aller, L. H., \& Czyzak, S. J. 1983, ApJS, 51, 211

Aller, L. H., Keyes, C. D., \& Feibelman, W. 1988, PASP, 100, 192

Baker, J. G., \& Menzel, D. H. 1938, ApJ, 88, 52

Barker, T. 1978, ApJ, 193, 209

Barker, T. 1983, ApJ, 267, 630 
Barker, T. 1986, ApJ, 308, 314

Blöcker, T. 1995, ApJ, 371, 217

Blöcker, T., \& Schönberner, D. 1990, A\&A, 240, L11

Boffi, F. R., \& Stanghellini, L. 1994, A\&A, 284, 248

Bohigas, J. 1994, A\&A, 288, 617

Brocklehurst, M. 1971, MNRAS, 153, 471

Buckley, D., \& Schneider, S. E. 1995, ApJ, 46, 279

Butler, S. E., Heil, T. G., \& Dalgarno, A. 1980, ApJ, 241, 442

Chengalur, J. N., Lewis, B. M., Eder, J., \& Terzian, Y. 1993, ApJS, 89, 189

Clegg, R. E. S., Harrington, J. P., \& Storey, P. J. 1986, MNRAS, 221, 61P

Clegg, R. E. S., Miller, S., Storey, P. J., \& Kisielius, R. 1999, A\&AS, 135, 359

Corradi, R. L. M., Goncalves, D. R., Villaver, E., Mampaso, A., \& Perinotto, M. 2000, ApJ, 542, 861

Cuesta, J. P., \& Phillips, J. P. 1999, AJ, 117, 974

Cuesta, J. P., \& Phillips, J. P. 2000, ApJ, 543, 754

Curtis, H. D. 1918, Publ. Lick Obs. 13, 55

David, P., Le Squeren, A. M., \& Sivagnanam, P. 1993, A\&A, 277,474

de Freitas Pacheco, J. A., Maciel, W. J., \& Costa, R. D. D. 1992, A\&A, 261, 579

de Oliveira-Abans, M., \& Faundez-Abans, M. 1991, Rev. Mex. Astron. Astrophys., 22, 3

De Robertis, M. M., Osterbrock, D. E., \& McKee, C. F. 1985, ApJ, 293, 459

Dopita, M. A., Lawrence, C. J., Ford, H. C., \& Webster, B. L. 1985, ApJ, 296, 390

Dopita, M. A., Vassiliadis, E., Meatheringham, S. J., et al. 1996, ApJ, 460, 320

Ferland, G. J., Korista, K. T., Verner, D. A., et al. 1998, PASP, 110, 761

Flower, D. R. 1969, MNRAS, 146, 171

Frank, A. 1999, NewA Rev., 43, 31

Gathier, R. 1987, A\&AS, 71, 245

Gathier, R., \& Pottasch, S. R. 1988, A\&A, 197, 266

Gathier, R., \& Pottasch, S. R. 1989, A\&A, 209, 369

Gathier, R., Pottasch, S. R., \& Pel, J. W. 1986, A\&A, 157, 171

Gorny, S. K., Schwarz, H. E., Corradi, R. L. M., \& Van Winckel, H. 1999, A\&AS, 136, 145

Gorny, S. K., Stasinska, G., \& Tylenda, R. 1997, A\&A, 318, 256

Greig, W. E. 1972, A\&A, 18, 70

Gruenwald, R., \& Viegas, S. M. 1995, A\&A, 303, 535

Habing, H. J. 1996, A\&ARv, 7, 97

Harrington, J. P., \& Marionni, P. A. 1976, ApJ, 206, 458

Hummer, D. G., \& Seaton, M. J. 1964, MNRAS, 127, 217

Hummer, D. G., \& Storey, P. J. 1987, MNRAS, 224, 801

Hyung, S., \& Aller, L. H. 1996, MNRAS, 278, 551

Iben, I. Jr. 1984, ApJ, 277, 333

Jacoby, G. H., \& Kaler, J. B. 1989, AJ, 98, 1662

Kastner, J. H., Weintraub, D. A., Gatley, I., Merrill, K. M., \& Probst, R. G. 1996, ApJ, 462, 777

Keenan, F. P., Aller, L. H., Bell, K. L., et al. 1999, MNRAS, 304, 27

Kingsburgh, R. L., \& Barlow, M. J. 1992, MNRAS, 257, 317

Kohoutek, L. 2000, Abhandlungen aus der Hamburger Sternwarte, XII

Kohoutek, L., \& Martin, W. 1981, A\&AS, 44, 325

Köppen, J., Acker, A., \& Stenholm, B. 1991, A\&A, 248, 197

Kovacs, G. 2000, A\&A, 363, L1
Liu, X.-W., Storey, P. J., Barlow, M. J., et al. 2000, MNRAS, 312,58

Maciel, W. J., Faundez-Abans, M., \& de Oliveira-Abans, M. 1986, RMxAA, 12, 233

Manchado, A., Guerrero, M. A., Stanghellini, L., \& SerraRicart, M. 1996, The IAC Morphological Catalog of Northern Planetary Nebulae, IAC

Marten, H., \& Szczerba, R. 1997, A\&A, 325, 1132

Martin, W. 1981, A\&A, 98, 328

Mathis, J. S., Torres-Peimbert, S., \& Peimbert, M. 1998, ApJ, 495,328

Mavromatakis, F., Papamastorakis, J., \& Paleologou, E. V. 2001, A\&A, 374, 280

Meatheringham, S. J., Wood, P. R., \& Faulkner, D. J. 1988, ApJ, 334, 862

O'Dell, C. R. 1962, ApJ, 135, 371

Osterbrock, D. E. 1989, Astrophysics of Gaseous Nebulae and Active Galactic Nuclei, Mill Valley, CA Univ. Sci.

Peimbert, M., \& Torres-Peimbert, S. 1971, ApJ, 168, 413

Pottasch, S. R. 1981, A\&A, 94, L13

Pottasch, S. R. 1983, in Planetary Nebulae, ed. D. R. Flower, IAU Symp., 103, 391

Pottasch, S. R. 1984, Planetary Nebulae, a Study of Late Stages of Stellar Evolution (Reidel, Dordrecht)

Ragazzoni, R., Cappellaro, E., Benetti, S., Turatto, M., \& Sabbadin, F. 2001, A\&A, 369, 1088

Reay, N. K., Pottasch, S. R., Atherton, P. D., \& Taylor, K. 1984, A\&A, 137, 113

Reay, N. K., \& Worswick, S. P. 1977, MNRAS, 179, 317

Sabbadin, F. 1986, A\&A, 160, 31

Sabbadin, F., Cappellaro, E., Benetti, S., Turatto, M., \& Zanin, C. 2000a, A\&A, 355, 688

Sabbadin, F., Benetti, S., Cappellaro, E., \& Turatto, M. 2000b, A\&A, 361, 1112

Sahai, R., \& Trauger, J. T. 1998, AJ, 116, 1357

Schmidt-Voigt, M., \& Köppen, J. 1987, A\&A, 174, 211

Schönberner, D. 1979, A\&A, 79, 108

Schönberner, D. 1981, A\&A, 103, 119

Schönberner, D. 1983, ApJ, 272, 708

Schwarz, H. E., Corradi, R. L. M., \& Melnick, J. 1992, A\&AS, 96,23

Seaton, M. J. 1979, MNRAS, 187, 73P

Shaw, R. A., \& Kaler, J. B. 1989, ApJS, 69, 495

Shields, G. A., Aller, L. H., Keyes, C. D., \& Czyzak, S. J. 1981, ApJ, 248, 569

Shields, G. A., Dalgarno, A., \& Sternberg, A. 1983, PhRva, 28, 2137

Sjouwerman, L. O., van Langevelde, H. J., Winnberg, A., \& Habing, H. J. 1998, A\&AS, 128, 35

Soker, L., \& Rappaport, S. 2001, ApJ, 557, 256

Spitzer, L. Jr. 1978, Physical Processes in the Interstellar Medium (John Wiley \& Sons, New York)

Stanghellini, L., Corradi, R. L. M., \& Schwarz, H. E. 1993, A\&A, 276, 463

Stasinska, G., Tylenda, R., Acker, A., \& Stenholm, B. 1992, A\&A, 266, 486

Tylenda, R. 1983, A\&A, 126, 299

Tylenda, R. 1986, A\&A, 156, 217

Tylenda, R., \& Stasinska, G. 1994, A\&A 288, 897

Vassiliadis, E., \& Wood, P. R. 1993, ApJ, 413, 641

Vassiliadis, E., \& Wood, P. R. 1994, ApJS, 92, 125

Weedman, D. W. 1968, ApJ, 153, 49

Williams, R. E. 1973, MNRAS, 164, 111

Wilson, O. C. 1950, ApJ, 111, 279

Wood, P. R., \& Faulkner, D. J. 1986, ApJ, 307, 659

Zhang, C. Y. 1995, ApJS, 98, 659 